\title{
Sharing Logistics Service Supply Chain with Revenue-Sharing vs. Cost-Sharing Contracts
}

\author{
Ying Luo, ${ }^{1}$ Qiang Wei $\mathbb{D}^{2,3}$ Xinyu Gou, ${ }^{4}$ Dai Dai, ${ }^{5}$ and Yiran Zhou ${ }^{4}$ \\ ${ }^{1}$ School of Business, Hunan University of Science and Technology, Xiangtan 411100, China \\ ${ }^{2}$ School of Management and Economics, University of Electronic Science and Technology of China, Chengdu 611731, China \\ ${ }^{3}$ School of Statistics, Southwestern University of Finance and Economics, Chengdu 611130, China \\ ${ }^{4}$ School of Business Administration, Southwestern University of Finance and Economics, Chengdu 611130, China \\ ${ }^{5}$ School of Economic Mathematics, Southwestern University of Finance and Economics, Chengdu 611130, China \\ Correspondence should be addressed to Qiang Wei; wq_research@126.com
}

Received 26 August 2020; Revised 30 November 2020; Accepted 20 December 2020; Published 16 January 2021

Academic Editor: Miguel A. Salido

Copyright (c) 2021 Ying Luo et al. This is an open access article distributed under the Creative Commons Attribution License, which permits unrestricted use, distribution, and reproduction in any medium, provided the original work is properly cited.

\begin{abstract}
The purpose of this study is to explore the design of equity cooperation mechanism in the sharing logistics service supply chain. This study designs a two-echelon logistics service supply chain composed of an urban joint distribution company and $N$ logistics companies. The urban joint distribution company is jointly established by $N$ logistics companies based on specific shares of equity investment. We establish sharing logistics service supply chain models under revenue-sharing or cost-sharing contracts. Revenuesharing factor or cost-sharing factor is the equity cooperation parameter. When the members of the supply chain choose to cooperate in revenue-sharing or cost-sharing mechanism, not all cooperation scenarios considered in the study can achieve Pareto improvement of the total profit of the supply chain, but at least one situation can achieve Pareto improvement. This study provides feasible solutions for logistics companies to join the sharing logistics service platform and provides a reference for the operation of a joint distribution platform established by logistics companies. New results and managerial insights are derived by the sharing logistics service supply chain with revenue-sharing vs cost-sharing contracts, which enriches the interfaces of the operation of the sharing logistics service supply chain.
\end{abstract}

\section{Introduction}

The development of the sharing economy has provided great convenience to our lives, such as sharing cars and sharing bicycles to ease travel problems. At present, the impact of COVID-19 has made the shared medical and shared education industries grow against the trend. It is predicted that China's sharing economy will account for $10 \%$ of GDP in 2020 , and it will reach $20 \%$ in 2025 [1]. The sharing economy was written into China's "Government Work Report" from 2017 to 2020, which shows that China attaches great importance to the development of the sharing economy.

In recent years, typical business models of shared logistics such as shared distribution, shared warehousing, and shared logistics dedicated lines have been favored by the capital market. Sichuan Tongyue Supply Chain Management
Co., Ltd. (Tongyue), Dazhou Dayun Logistics Co., Ltd. (Dayun), Sichuan Wangyong Logistics Co., Ltd. (Wangyong), and Sichuan Yusheng Logistics Development Co., Ltd. (Yusheng) made a joint investment in Sichuan Yuanyao Supply Chain Management Co., Ltd. (Yuanyao) in 2018 in Sichuan, China. The shareholder units of Yuanyao are mainly engaged in the logistics transportation business from Dazhou, Yibin, and Xichang to Chengdu. Yuanyao's shareholder units have effectively cooperated to develop the feasibility of urban joint distribution from 2018 to 2019.

Some questions about sharing logistics service supply chain deserve attention, such as why do companies with different logistics service capabilities choose to cooperate? Why do many logistics companies choose to cooperate to build a distribution platform in urban distribution? How do logistics companies with different logistics services and 
logistics capabilities solve the "free-riding" behavior of small enterprises in cooperation?

Motivated by these questions, we propose a new operating mechanism and design a two-echelon service supply chain for a common distribution logistics system composed of urban joint distribution companies and $N$ logistics companies. Meanwhile, the two-echelon service supply chain is based on equity cooperation. What we need to decide is whether to choose revenue-sharing or cost-sharing contracts.

The contributions of our study are as follows. First, we study the $1-N$ supply chain structure, which is more realistic. Second, we introduce the concept of sharing economy into the field of urban distribution and design a path for cooperation and sharing in the study. Third, we summarize and refine the business-based equity cooperation phenomenon that often occurs in the modern logistics industry after field research on many logistics companies and e-commerce logistics companies. Based on this, we propose a joint distribution cooperation mechanism and combine the core parameters of supply chain contract with equity design.

The study is organized as follows. Section 2 presents the literature review. Section 3 introduces the research problem hypothesis and model setting. Section 4 shows the benchmark model. The analysis of the optimal strategy of revenuesharing and cost-sharing contract is evaluated in Sections 5 and 6. Section 7 presents the case analysis. Conclusions are summarized in Section 8.

\section{Literature Review}

The study focuses on logistics service supply chain and sharing mechanism. The research on the sharing mechanism in the field of operation management mainly has two directions: one is the research on the business model of sharing economy, such as shared bikes and cars, and the other is to study the sharing and cooperation mechanism in operation management, such as information-sharing, capacitysharing, revenue-sharing, and cost-sharing.

2.1. Logistics Service Supply Chain. Logistics service supply chain is composed of a logistics service integrator and some functional logistics service providers [2-5]. Liu et al. [6] study the fairest revenue-sharing coefficient when logistics service integrators and functional logistics service providers implement revenue-sharing contracts under random demand. Liu et al. [7] discuss the order distribution of the logistics service supply chain. Liu et al. [8] consider how a two-echelon logistics service supply chain can minimize the average unit operating cost of logistics service integrators and maximize the average satisfaction of logistics service providers due to their capacities and time constraints. The influence of loss aversion preference on logistics service supply chain members' decision-making is discussed by Liu et al. [9]. Shen et al. [10] focus on the trade-off between transportation time and cost in the global supply chain and provide insights on how to manage logistics services. Wang et al. [11] investigate the selection of service providers and order allocation in different processes for mass customized logistics service models and design an improved genetic algorithm to solve.

2.2. Business Model of Sharing Economy. The sharing economy, in which ordinary consumers also act as sellers, is attracting great interest from scholars and practitioners. The rapid growth of this emerging economic form and the emergence of several big brands such as Uber and Airbnb raise many interesting questions to be studied [12]. Transaction costs in the shared market have a nonmonotonic effect on company profits, consumer surplus, and social welfare. When a company strategically chooses retail prices, it is a win-win situation for consumers to share products with high marginal costs, but it may be a lose-lose situation for them if they share products with low marginal costs [13].

More than 400 cities around the world have deployed or plan to deploy bicycle sharing systems. However, the factors driving their use and the amount of rebalancing they require are unclear [14]. In view of the increasing importance of bike sharing systems, Caggiani et al. [15] propose a new integrated dynamic bike redistribution method. With the rapid development of bicycle sharing, more and more issues related to it have been studied: such as bicycle sharing stations based on maximum coverage location, factors affecting bicycle sharing members, and its economic contribution to the sustainability and efficient operation of the city [16].

\subsection{Revenue-Sharing and Cost-Sharing Contracts.} Revenue-sharing contracts are popular in the cassette tape rental industry [17]. Liu et al. [6] show that related concepts of sharing mechanism can be introduced into the study of logistics service supply chain management. Li et al. [18] propose that consignment contract under revenue-sharing has been widely used in many industries, especially in the online retail market. Pan et al. [19] show that the revenuesharing contract can improve supply chain performance. Kimms and Çetiner [20] propose a nucleolus-based revenuesharing cooperative game strategy based on operational optimization to solve the problem of equitable income distribution in airline alliances. Van der Rhee et al. [21] point out that although there have been many studies on revenuesharing contracts, the application rate of their research results in the industry is not high. Zhang et al. [22] design the revenue-sharing and cooperative investment contract to coordinate the supply chain. Wei et al. [23] reveal a joint optimal decision-making strategy that involves revenuesharing and cooperative investment contracts based on an order flow proportion (OFP) and a revenue-sharing factor (RSF) and find that an OFP system offers the best solution in designing revenue-sharing contracts based on RSFs. Costsharing contract can improve profits of green food supply chain members $[24,25]$. Revenue-sharing and cost-sharing contracts are widely used together in the researches [26-35]. 
The works that are of particular relevance to our study are by Zhang et al. [22], Yang and Chen [31], Wei et al. [23], and $\mathrm{Yu}$ et al. [33]. As only the implicit solution of the optimal decision can be obtained, the optimal decision is finally presented through numerical examples. Similar to Zhang et al. [22], our optimal decision can only be obtained by an implicit solution, and finally, a numerical example is used to choose a cooperative contract. Wei et al. [23] discuss an omnichannel supply chain structure in the joint distribution logistics cooperation model, but we design a mechanism of revenue-sharing and cost-sharing based on equity cooperation. Yang and Chen [31] and Yu et al. [33] both discuss whether supply chain members choose sharing of revenue, cost, or both in emission reduction cooperation. However, we consider whether urban joint distribution company and many logistics companies should choose revenue-sharing contract or cost-sharing contract to cooperate to improve the performance of logistics service supply chain.

\section{Model Description and Assumptions}

We design a two-echelon omnichannel service supply chain of a joint distribution logistics system composed of an urban joint distribution company and $N$ logistics companies, in which logistics companies only operate the intercity logistics transportation, and the urban distribution service is outsourced to the urban joint distribution company. Unlike the traditional urban logistics operation model, the urban joint distribution company is jointly established by $N$ logistics companies according to certain proportions of equity investment. In this study, the proportion of equity investment is uniquely determined by the proportion of revenue-sharing or cost-sharing. We summarize all notations as follows.

Similar to the assumption about the demand function by Ma et al. [36] and Wei et al. [23], we assume that the demand function of logistics company $i$, as shown in the following equation:

$$
\begin{aligned}
x_{i}= & \underbrace{\lambda_{i} d-\alpha p_{i}+\beta \sum_{\substack{j=1 \\
j \neq i}}^{n} p_{j}+\varepsilon,}_{\widehat{x}_{i}} \\
& \quad i=1,2,3, \ldots, n, \text { where } i=1,2,3, \ldots, n .
\end{aligned}
$$

In equation (1), $\lambda_{i}$ represents the market share of logistics company $i, \lambda_{i} \in[0,1]$, and $\sum_{i=1}^{n} \lambda_{i}=1$. The demand $x_{i}$ of logistics company $i$ is negatively correlated with the unit service price $p_{i}$ provided by logistics company $i$ and positively correlated with the unit service price $p_{j}$ of all other logistics company $j . \widehat{x}_{i}$ is the average value of the market demand of logistics company $i$. $\varepsilon$ is the disturbance term of the random market demand, which obeys uniform distribution and represents other influencing factors of demand uncertainty and satisfiese $\sim U[-h, h]$, where $h$ is an interval value of this uniform distribution $E(\varepsilon)=0$.
As an independent service entity, the urban joint distribution company provides all logistics companies with urban joint distribution services. The profit function of the urban joint distribution company is as follows:

$$
\pi_{s}=w \sum_{i=1}^{n} q_{i}-\left[c \sum_{i=1}^{n} q_{i}+e\left(\sum_{i=1}^{n} q_{i}\right)^{2}\right] .
$$

In equation (2), the operating cost of the urban joint distribution company not only includes unit service cost but also includes nonscale economy costs $e\left(\sum_{i=1}^{n} q_{i}\right)^{2}$, where $e\left(\sum_{i=1}^{n} q_{i}\right)^{2}$ is the non-scale economic cost, which is mainly incurred due to the increase in costs caused by management capabilities and user service access [37, 38].

The profit equals the revenue minus the costs, so the profit function of logistics company $i$ is as follows:

$$
\begin{aligned}
\pi_{i}= & \int_{\widehat{x_{i}}-h}^{q_{i}}\left(p_{i}-w-c_{i}\right) x_{i} f\left(x_{i}\right) \mathrm{d} x_{i} \\
& +\int_{q_{i}}^{\widehat{x_{i}}+h}\left(p_{i}-w-c_{i}\right) q_{i} f\left(x_{i}\right) \mathrm{d} x_{i} .
\end{aligned}
$$

There will be an oversupply or undersupply of logistics services due to the asymmetry of market information and the inaccuracy of market demand forecasts in practice. Therefore, overcapacity cost and undercapacity cost caused by random fluctuations in market demand will be considered in this study.

Overcapacity cost is as follows:

$$
O_{i}(\vec{p}, \vec{q})=\int_{\widehat{x}_{i}}-h q_{i}(w-v)\left(q_{i}-x_{i}\right) f\left(x_{i}\right) \mathrm{d} x_{i},
$$

where $O_{i}(\vec{p}, \vec{q})$ is the cost of providing logistics service at a level lower than the market price due to excess logistics service capacity and $v$ is the unit residual value of logistics service in equation (4). The specific connotation of services and general physical products residual value is different. The unit residual value of general physical products may be the value that can be generated after the physical product is used for other purposes or processed; the residual value of logistics service capabilities represents the profit obtained by temporarily providing the logistics service capacity to other social customers at a level lower than the market price.

Undercapacity cost is as follows:

$$
U_{i}(\vec{p}, \vec{q})=\int_{q_{i}}^{\widehat{x_{i}}+h} k\left(x_{i}-q_{i}\right) f\left(x_{i}\right) \mathrm{d} x_{i},
$$

where $U_{i}(\vec{p}, \vec{q})$ is the cost incurred by missing market opportunities due to insufficient supply of logistics service capabilities.

The assumption is as follows:

A1: there is positive market demand, that is, $d>0$.

A2: the unit service cost of urban joint distribution company is significantly higher than the diseconomies of scale, and it is greater than zero, that is, $c \gg e>0$.

A3: $k>v \geq 0$. 


\section{Centralized and Decentralized Service Supply Chain: The Benchmark}

We study the optimal decision-making problem of centralized service supply chain and decentralized service supply chain of the urban joint distribution cooperation model.
4.1. Centralized Service Supply Chain. The centralized service supply chain takes the total profit of the supply chain as the objective function and the members of the supply chain jointly bear the risk loss incurred due to the oversupply or undersupply of logistics services. Therefore, the total profit of the centralized service supply chain can be expressed as follows, and the detail of the following equation is shown in Appendix (A.1):

$$
\begin{aligned}
\pi_{\mathrm{sc}}^{c}= & \sum_{i=1}^{n}\left\{\frac{\left(p_{i}-c_{i}-v\right)}{4 h}\left[q_{i}^{2}-\left(\widehat{x}_{i}-h\right)^{2}\right]+\frac{v q_{i}}{2 h}\left(q_{i}-\widehat{x}_{i}+h\right)+\frac{q_{i}\left(p_{i}-c_{i}+k\right)}{2 h}\left(\widehat{x}_{i}+h-q_{i}\right)-\frac{k}{4 h}\left[\left(\widehat{x}_{i}+h\right)^{2}-q_{i}^{2}\right]\right\} \\
& -c \sum_{i=1}^{n} q_{i}-e\left(\sum_{i=1}^{n} q_{i}\right)^{2} .
\end{aligned}
$$

By analyzing equation (6), we obtain Proposition 1.

Proposition 1. The optimal solution for the centralized service supply chain satisfies the following equation: $\left(\vec{p}^{*}, \vec{q}^{*}\right)=\arg \max \left\{\pi_{s c}^{c}\left(\Lambda_{0}^{c}\right), \pi_{s c}^{c}\left(\Lambda_{\vec{p}}^{c}, \Lambda_{\vec{q}}^{c}\right)\right\}$, where $\Lambda_{0}^{c}$ is the boundary of the feasible region of the decision variable and $\left(\Lambda_{\vec{p}}^{c}, \Lambda_{\vec{q}}^{c}\right)$ denotes all the maximum points of the decision function in the feasible region of the decision variable. It also satisfies the following system of equations:

$$
\left\{\begin{array}{l}
-\frac{q_{i}^{* 2}}{4 h}+\frac{q_{i}^{*}}{2 h}\left(\hat{x}_{i}^{*}+h+\alpha v-\alpha p_{i}^{*}+\alpha c_{i}-\alpha k\right)-\frac{\left(\hat{x}_{i}^{*}-h^{2}\right)}{4 h}+\frac{\alpha\left(p_{i}^{*}-c_{i}-v\right)\left(\hat{x}_{i}^{*}-h\right)}{2 h}+\frac{\alpha k\left(\hat{x}_{i}^{*}+h\right)}{2 h} \\
\sum_{\substack{j=1 \\
j \neq 1}}^{n}\left[-\frac{\beta\left(p_{j}^{*}-c_{j}-v\right)\left(\hat{x}_{j}^{*}-h\right)}{2 h}+\frac{\beta q_{j}^{*}\left(p_{j}^{*}-c_{j}+k-v\right)}{2 h}-\frac{\beta k\left(\hat{x}_{j}^{*}+h\right)}{2 h}\right]=0, \\
\frac{\left(\hat{x}_{i}^{*}-q_{i}^{*}\right)\left(p_{j}^{*}=c_{i}-v+k\right)}{2 h}+\frac{p_{i}^{*}-c_{i}+k+v}{2}-c-2 e \sum_{j=1}^{n} q_{j}^{*}=0, \\
\Lambda_{\vec{p}}^{c}=\left\{p_{1}^{*}, p_{2}^{*}, \ldots, p_{n}^{*}\right\}, p_{i}^{*} \in \Lambda_{\vec{p}}^{c} ; \Lambda_{\vec{q}}^{c}=\left\{q_{1}^{*}, q_{2}^{*}, \ldots, q_{n}^{*}\right\}, q_{i}^{*} \in \Lambda_{\vec{q}}^{c} .
\end{array}\right.
$$

Proof. Refer to the Appendix.

We can derive from equation (7) that the decision variable is the order quantity (or logistics service price) of each logistics company, and as it is a multivariate cubic equation, it is difficult to solve for the explicit expression of the optimal decision, but the implicit expression can be derived through Proposition 1. After giving other corresponding parameters, we could simulate and present the optimal decision through numerical examples.

4.2. Decentralized Service Supply Chain. In the analysis of the decentralized supply chain, two scenarios are taken into consideration: urban joint distribution company bearing 
cost risk and logistics companies independently bearing cost risk. In the decentralized supply chain, the urban joint distribution company and logistics companies will pursue profit maximization.

4.2.1. Logistics Companies Bear Cost Risks. The optimal decision of the urban joint distribution company is as follows:

$$
\frac{d \pi_{s}^{d 1}}{d q_{i}}=w-\left(c+2 e \sum_{j=1}^{n} q_{j}\right)=0, \text { that is, } w=c+2 e \sum_{j=1}^{n} q_{j}
$$

The profit of the logistics company $i$ is as follows, and the detail of the following equation is shown in (A.2):

$$
\begin{aligned}
\pi_{i}^{d 1}= & \frac{\left(p_{i}-c_{i}-v\right)}{4 h}\left[q_{i}^{2}-\left(\widehat{x}_{i}-h\right)^{2}\right]+\frac{v q_{i}}{2 h}\left(q_{i}-\widehat{x}_{i}+h\right)+\frac{q_{i}\left(p_{i}-c_{i}+k\right)}{2 h}\left(\widehat{x}_{i}+h-q_{i}\right)-\frac{k}{4 h}\left[\left(\widehat{x}_{i}+h\right)^{2}-q_{i}^{2}\right] \\
& -c q_{i}-2 e q_{i} \sum_{j=1}^{j=n} q_{j} .
\end{aligned}
$$

Proposition 2. In a decentralized service supply chain where logistics companies independently bear operational cost risks, the optimal solution satisfies the following system of equations $\left(p_{i}^{*}, q_{i}^{*}\right)=\arg \max \left\{\pi_{i}^{d 1}\left(\Lambda_{0}^{d 1}\right), \pi_{i}^{d 1}\left(\Lambda_{p_{i}}^{d 1}, \Lambda_{q_{i}}^{d 1}\right)\right\}$, where $\Lambda_{0}^{d 1}$ is the boundary of the feasible region of the decision variable and $\left(\Lambda_{p_{i}}^{d 1}, \Lambda_{q_{i}}^{d 1}\right)$ denotes all the maximum points of the decision function in the feasible region of the decision variable. It also satisfies the following system of equations:

$$
\left\{\begin{array}{l}
-\frac{q_{i}^{* 2}}{4 h}+\frac{q_{i}^{*}\left(\hat{x}_{i}^{*}+h\right)}{2 h}-\frac{\alpha q_{i}^{*}\left(p_{i}^{*}-c_{i}-v+k\right)}{2 h}-\frac{\left(\hat{x}_{i}^{*}-h\right)^{2}}{4 h}+\frac{\alpha\left(p_{i}^{*}-c_{i}-v\right)\left(\hat{x}_{i}^{*}-h\right)}{2 h}+\frac{\alpha k}{2 h}\left(\hat{x}_{i}^{*}+h\right)=0 \\
\frac{\left(p_{i}^{*}-c_{i}+k-v\right)\left(\hat{x}_{i}^{*}-q_{i}^{*}\right)}{2 h}+\frac{p_{i}^{*}-c_{i}+k+v}{2}-c-2 e \sum_{j=1}^{n} q_{j}^{*}-2 e q_{i}^{*}=0 \\
\Lambda_{p_{i}}^{d 1}=\left\{p_{i}^{*}\right\}, p_{i}^{*} \in \Lambda_{p_{i}}^{d 1} ; \Lambda_{q_{i}}^{d 1}=\left\{q_{i}^{*}\right\}, q_{i}^{*} \in \Lambda_{q_{i}}^{d 1} .
\end{array}\right.
$$

Proof. Refer to the Appendix.

As shown in equation (10), similar to the centralized optimal solution, the decision variable of the decentralized supply chain is the order quantity (or logistics service price) of each logistics company and it is a binary cubic equation system. It is difficult to solve for its explicit expression, but the implicit expression can be derived through Proposition 2 .

$$
\begin{aligned}
\pi_{s}^{d 2}= & w \sum_{i=1}^{n} q_{i}-\left[c \sum_{i=1}^{n} q_{i}+e\left(\sum_{i=1}^{n} q_{i}\right)^{2}\right] \\
& -\sum_{i=1}^{n}\left\{\frac{(w-v) q_{i}}{2 h}\left(q_{i}-\widehat{x}_{i}+h\right)-\frac{(w-v)}{4 h}\left[q_{i}^{2}-\left(\widehat{x}_{i}-h\right)^{2}\right]+\frac{k}{4 h}\left[\left(\widehat{x}_{i}+h\right)^{2}-q_{i}^{2}\right]-\frac{k q_{i}}{2 h}\left(\widehat{x}_{i}+h-q_{i}\right)\right\} .
\end{aligned}
$$

4.2.2. Urban Joint Distribution Company Bears Cost Risks. The profit function of the urban joint distribution company is derived as follows, and the detail of the following equation is shown in Appendix (A.3): 
We solve first derivatives of the function $\pi_{s}^{d 2}$ with respect to the variable $q_{i}$ and equate it to 0 to obtain equation (12), and the detail of the first derivatives of the function $\pi_{s}^{d 2}$ with respect to the variable $q_{i}$ is shown in (A.4):

$$
w=\frac{2 h\left(c+2 e \sum_{j=1}^{n} q_{j}\right)+(k-v)\left(q_{i}-\widehat{x}_{i}\right)-h(k+v)}{\widehat{x}_{i}+h-q_{i}} .
$$

As $\forall i, j \in N, i \neq j$, we can obtain $q_{i}-\widehat{x}_{i}=q_{j}-\widehat{x}_{j}$. Thus, assuming $q_{1}-\widehat{x}_{1}=q_{2}-\widehat{x}_{2}=q_{3}-\widehat{x}_{3}=\cdots=q_{n}-\widehat{x}_{n}=Q \epsilon$ $[-h, h]$, then we have $\widehat{x}_{i}=q_{i}-Q, i=1,2,3, \ldots, n \widehat{x}_{i}=\lambda_{i} d-$ $\alpha p_{i}+\beta \sum_{\substack{j=1 \\ j \neq i}}^{n} p_{j}$ can be obtained from equation (1). If $\sum_{i=1}^{n} \widehat{x}_{i}=\sum_{i=1}^{n}\left(\lambda_{i} d-\alpha p_{i}+\beta \sum_{\substack{j=1 \\ j \neq i}}^{n} p_{j}\right)=d-\alpha \sum_{i=1}^{n} p_{i}+(n-$

1) $\beta \sum_{i=1}^{n} p_{i}=\sum_{i=1}^{n} q_{i}-n Q$, then we obtain the expression of $p_{i}$, as the following equation:

$$
p_{i}=\frac{\lambda_{i} d+Q-q_{i}}{\alpha+\beta}+\frac{\sum_{i=1}^{n} q_{i}-n Q-d}{(\alpha+\beta)(n-1-(\alpha / \beta))} .
$$

Equations (12) and (13) are the optimal decisions for the urban joint distribution company. Combining equations (12) and (13), we can obtain the profit of logistics company $i$ as follows and derive Proposition 3, and the detail of the following equation is shown in Appendix (A.5):

$$
\begin{aligned}
\pi_{i}^{d 2}= & \frac{1}{4 h}\left[\frac{\lambda_{i} d+Q-q_{i}}{\alpha+\beta}+\frac{\sum_{i=1}^{n} q_{i}-n Q-d}{(\alpha+\beta)(n-1-(\alpha / \beta))}-\frac{2 h\left(c+2 e \sum_{j=1}^{n} q_{j}\right)+(k-v) Q-h(k+v)}{h-Q}-c_{i}\right] \\
& {\left[-q_{i}^{2}-\left(q_{i}-Q-h\right)^{2}+2 q_{i}\left(q_{i}-Q+h\right)\right] . }
\end{aligned}
$$

Proposition 3. The optimal solution in the decentralized supply chain when the urban joint distribution company bearing cost risks satisfies the following equation: $\left(q_{i}^{*}, Q^{*}\right)=\arg \max \left\{\pi_{i}^{d 2}\left(\Lambda_{0}^{d 2}\right), \pi_{i}^{d 2}\left(\Lambda_{q_{i}}^{d 2}, \Lambda_{Q}^{d 2}\right)\right\}$, where $\Lambda_{0}^{d 2}$ is the boundary of the feasible region of the decision variable and $\left(\Lambda_{q_{i}}^{d 2}, \Lambda_{Q}^{d 2}\right)$ denotes all the maximum points of the decision function in the feasible region of the decision variable. It also satisfies the following system of equations:

$$
\left\{\begin{array}{l}
\frac{\lambda_{i} d+Q^{*}-q_{i}^{*}}{\alpha+\beta}+\frac{\sum_{j=1}^{n} q_{j}^{*}-n Q^{*}-d}{(\alpha+\beta)(n-1-(\alpha / \beta))}-\frac{2 h\left(c+2 e \sum_{j=1}^{n} q_{j}^{*}\right)+(k-v) Q^{*}-h(k+v)}{h-Q^{*}}-c_{i} \\
+\frac{-q_{i}^{* 2}-\left(q_{i}^{*}-Q^{*}-h\right)^{2}+2 q_{i}^{*}\left(q_{i}^{*}-Q^{*}+h\right)}{4 h} \times\left[-\frac{1}{\alpha+\beta}+\frac{1}{(\alpha+\beta)(n-1-(\alpha / \beta))}-\frac{4 h e}{h-Q^{*}}\right]=0, \\
\Lambda_{q_{i}}^{d 2}=\left\{q_{i}^{*}\right\}, q_{i}^{*} \in \Lambda_{q_{i}}^{d 2} ; \Lambda_{Q}^{d 2}=\left\{Q^{*}\right\}, Q^{*} \in[-h, h] .
\end{array}\right.
$$

Proof. Refer to the Appendix.

Similar to the centralized optimal solution, the implicit expression can be derived through Proposition 3. However, we could see that the dimensions of the decision-making variables in Proposition 3 are lower than that in Propositions 1 and 2, which means the decision complexity has been reduced. After giving other corresponding parameters, we could simulate and present the optimal decision through numerical examples.

\section{Revenue-Sharing Cooperative Decision}

According to the analysis of the decentralized service supply chain, it is feasible for the urban joint distribution company and each logistics company to independently bear the cost risk. Therefore, in revenue-sharing cooperation, these two situations are also considered. At the same time, the revenue-sharing strategies of an order flow proportion type and a factor combination type are analyzed, respectively, in each case of cost-sharing.

5.1. Revenue-Sharing When Logistics Company Bears Cost Risk. We can obtain the optimal decision of the urban joint distribution company as follows:

$$
w=c+2 e \sum_{j=1}^{n} q_{j}
$$

5.1.1. Revenue-Sharing under Order Flow Proportion When Logistics Companies Bear Cost Risk. Each logistics company 
independently bears its own operating cost risk and determines its revenue-sharing strategy according to the proportion of its order flow contribution. The proportion of order flow contribution for logistics company $i$ is as follows:

$$
\left(\frac{q_{1}}{\sum_{j=1}^{n} q_{j}}, \frac{q_{2}}{\sum_{j=1}^{n} q_{j}}, \frac{q_{3}}{\sum_{j=1}^{n} q_{j}}, \ldots, \frac{q_{n}}{\sum_{j=1}^{n} q_{j}}\right) .
$$

The profit of logistics company $i$ in an order flow proportion revenue-sharing supply chain when logistics companies bearing cost risks is as follows, and the detail of the following equation is shown in Appendix (A.6):

$$
\begin{aligned}
\pi_{i}^{r f 1}= & \frac{\left(p_{i}-c_{i}-v\right)}{4 h}\left[q_{i}^{2}-\left(\hat{x}_{i}-h\right)^{2}\right]+\frac{v q_{i}}{2 h}\left(q_{i}-\hat{x}_{i}+h\right)+\frac{q_{i}\left(p_{i}-c_{i}+k\right)}{2 h}\left(\hat{x}_{i}+h-q_{i}\right)-\frac{k}{4 h}\left[\left(\hat{x}_{i}+h\right)^{2}-q_{i}^{2}\right] \\
& -c q_{i}-e q_{i} \sum_{j=1}^{n} q_{j} .
\end{aligned}
$$

By analyzing equation (18), we can obtain Proposition 4.

Proposition 4. The optimal solution in an order flow proportion revenue-sharing supply chain when logistics companies bearing cost risks satisfies the following equation: $\left(p_{i}^{*}, q_{i}^{*}\right)=\arg \max \left\{\pi_{i}^{r f 1}\left(\Lambda_{0}^{r f 1}\right), \pi_{i}^{r f 1}\left(\Lambda_{p_{i}}^{r f 1}, \Lambda_{q_{i}}^{r f 1}\right)\right\}$, where
$\Lambda_{0}^{r f 1}$ is the boundary of the feasible region of the decision variable and $\left(\Lambda_{p_{i}}^{r f 1}, \Lambda_{q_{i}}^{r f 1}\right)$ denotes all the maximum points of the decision function in the feasible region of the decision variable. It also satisfies the following system of equations:

$$
\left\{\begin{array}{l}
-\frac{q_{i}^{* 2}}{4 h}+\frac{q_{i}^{*}\left(\hat{x}_{i}^{*}+h\right)}{2 h}-\frac{\alpha q_{i}^{*}\left(p_{i}^{*}-c_{i}-v+k\right)}{2 h}-\frac{\left(\hat{x}_{i}^{*}-h\right)^{2}}{4 h}+\frac{\alpha\left(p_{i}^{*}-c_{i}-v\right)\left(\hat{x}_{i}^{*}-h\right)}{2 h}+\frac{\alpha k}{2 h}\left(\hat{x}_{i}^{*}+h\right)=0 \\
\frac{\left(p_{i}^{*}-c_{i}+k-v\right)\left(\hat{x}_{i}^{*}-q_{i}^{*}\right)}{2 h}+\frac{p_{i}^{*}-c_{i}+k+v}{2}-c-e \sum_{j=1}^{n} q_{j}^{*}-e q_{i}^{*}=0, \\
\Lambda_{p_{i}}^{r f 1}=\left\{p_{i}^{*}\right\}, p_{i}^{*} \in \Lambda_{p_{i}}^{r f 1} ; \Lambda_{q_{i}}^{r f 1}=\left\{q_{i}^{*}\right\}, q_{i}^{*} \in \Lambda_{q_{i}}^{r f 1} .
\end{array}\right.
$$

Proof. Refer to the Appendix.

Similar to the centralized and the decentralized optimal solution, the implicit expression of the optimal decision variable can be derived through Proposition 4. The implicit expression in Proposition 4 is similar to that in Proposition 2 because the logistics company bears the cost risks in both the situations. After presenting the other corresponding parameters, we could present the optimal decision through numerical examples.

5.1.2. Revenue-Sharing under Factor Combination When Logistics Companies Bear Cost Risk. The shared factor combination coefficient for logistics company $i$ is as follows:

$$
\begin{aligned}
& \left(\gamma_{1}, \gamma_{2}, \gamma_{3}, \ldots, \gamma_{n}\right), \quad \forall \gamma_{i} \in[0,1], \\
& \sum_{i=1}^{n} \gamma_{i}=1 .
\end{aligned}
$$

Then, we can obtain $\pi_{i}^{r r 1}$ as follows, and the detail of the following equation is shown in Appendix (A.7):

$$
\begin{aligned}
\pi_{i}^{r r 1}= & \frac{\left(p_{i}-c_{i}-v\right)}{4 h}\left[q_{i}^{2}-\left(\widehat{x}_{i}-h\right)^{2}\right]+\frac{v q_{i}}{2 h}\left(q_{i}-\widehat{x}_{i}+h\right) \\
& +\frac{q_{i}\left(p_{i}-c_{i}+k\right)}{2 h}\left(\widehat{x}_{i}+h-q_{i}\right)-\frac{k}{4 h}\left[\left(\widehat{x}_{i}+h\right)^{2}-q_{i}^{2}\right] \\
& -\left(c+2 e \sum_{j=1}^{n} q_{j}\right) q_{i} \\
& +\gamma_{i}\left\{\left(c+2 e \sum_{j=1}^{n} q_{j}\right) \sum_{i=1}^{n} q_{i}-\left[c \sum_{i=1}^{n} q_{i}+e\left(\sum_{i=1}^{n} q_{i}\right)^{2}\right]\right\} .
\end{aligned}
$$

By analyzing equation (21), we can obtain Proposition 5.

Proposition 5. The optimal solution in a factor combination revenue-sharing supply chain when logistics companies bearing cost risks satisfies the following equation: $\left(p_{i}^{*}, q_{i}^{*}\right)=\arg \max \left\{\pi_{i}^{r r 1}\left(\Lambda_{0}^{r r 1}\right), \pi_{i}^{r r 1}\left(\Lambda_{p_{i}}^{r r 1}, \Lambda_{q_{i}}^{r r 1}\right)\right\}$, where $\Lambda_{0}^{r r 1}$ is the boundary of the feasible region of the decision variable and $\left(\Lambda_{p_{i}}^{r r 1}, \Lambda_{q_{i}}^{r r 1}\right)$ denotes all the maximum points of the 
decision function in the feasible region of the decision variable.

It also satisfies the following system of equations:

$$
\left\{\begin{array}{l}
-\frac{q_{i}^{* 2}}{4 h}+\frac{q_{i}^{*}\left(\hat{x}_{i}^{*}+h\right)}{2 h}-\frac{\alpha q_{i}^{*}\left(p_{i}^{*}-c_{i}-v+k\right)}{2 h}-\frac{\left(\hat{x}_{i}^{*}-h\right)^{2}}{4 h}+\frac{\alpha\left(p_{i}^{*}-c_{i}-v\right)\left(\hat{x}_{i}^{*}-h\right)}{2 h}+\frac{\alpha k}{2 h}\left(\hat{x}_{i}^{*}+h\right)=0 \\
\frac{\left(p_{i}^{*}-c_{i}+k-v\right)\left(\hat{x}_{i}^{*}-q_{i}^{*}\right)}{2 h}+\frac{p_{i}^{*}-c_{i}+k+v}{2}-c-2 e \sum_{j=1}^{n} q_{j}^{*}-2 e q_{i}^{*}+2 \gamma_{i} e \sum_{j=1}^{n} q_{j}^{*}=0 \\
\Lambda_{p_{i}}^{r f 1}=\left\{p_{i}^{*}\right\}, p_{i}^{*} \in \Lambda_{p_{i}}^{r f 1} ; \Lambda_{q_{i}}^{r f 1}=\left\{q_{i}^{*}\right\}, q_{i}^{*} \in \Lambda_{q_{i}}^{r f 1} .
\end{array}\right.
$$

Proof. Refer to the Appendix.

Similar to the centralized and decentralized optimal solution, the implicit expression of the optimal decision variable can be derived through Proposition 5. We can see that the first subexpression both in the implicit expression of the Propositions 4 and 5 is the same one and there is no any difference, while the second subexpression is different at the $e q_{i}^{*}$ and $e q_{j}^{*}$ terms. We could present the optimal decision through numerical examples after giving other corresponding parameters.

5.2. Revenue-Sharing When the Urban Joint Distribution Company Bears Cost Risk. The profit of the urban joint distribution company is as follows:

$$
\begin{aligned}
\pi_{s}^{r 2}= & \underbrace{}_{\pi_{s}} \underbrace{n}_{i=1} q_{i}-\left[c \sum_{i=1}^{n} q_{i}+e\left(\sum_{i=1}^{n} q_{i}\right)^{2}\right] \\
& -\sum_{i=1}^{n}\left[O_{i}(\vec{p}, t \vec{q})+U_{i}(\vec{p}, \vec{q})\right] .
\end{aligned}
$$

Similar to the analysis of the profit and optimal decisionmaking of the urban joint distribution company, we can obtain the optimal decisions for the urban joint distribution company as follows:

$$
\begin{array}{cc}
w=\frac{2 h\left(c+2 e \sum_{j=1}^{n} q_{j}\right)+(k-v)\left(q_{i}-\widehat{x}_{i}\right)-h(k+v)}{\widehat{x}_{i}+h-q_{i}} & \forall i \in N \\
p_{i}=\frac{\lambda_{i} d+Q-q_{i}}{\alpha+\beta}+\frac{\sum_{i=1}^{n} q_{i}-n Q-d}{(\alpha+\beta)(n-1-(\alpha / \beta))}, & \forall i \in N
\end{array}
$$

Combined equations (24) and (25), we can obtain the profit of the logistics company $i$ as follows and Proposition 6 can be obtained.

5.2.1. Revenue-Sharing under Order Flow Proportion When the Urban Joint Distribution Company Bears Cost Risk. Logistics companies make decisions on their revenuesharing ratio according to their respective order flow contribution proportion, which is shown in the following equation:

$$
\left(\frac{q_{1}}{\sum_{j=1}^{n} q_{j}}, \frac{q_{2}}{\sum_{j=1}^{n} q_{j}}, \frac{q_{3}}{\sum_{j=1}^{n} q_{j}}, \ldots, \frac{q_{n}}{\sum_{j=1}^{n} q_{j}}\right),
$$

where $\pi_{i}^{r f 2}$ can be obtained as follows, and the detail of the following equation is shown in Appendix (A.8):

$$
\begin{aligned}
\pi_{i}^{r f 2}= & \left(\frac{\lambda_{i} d+Q-q_{i}}{\alpha+\beta}+\frac{\sum_{j=1}^{n} q_{j}-n Q-d}{(\alpha+\beta)(n-1-(\alpha / \beta))}-\frac{2 h\left(c+2 e \sum_{j=1}^{n} q_{j}\right)+(k-v) Q-h(k+v)}{h-Q}-c_{i}\right) \\
& \frac{\left[-q_{i}^{2}-\left(q_{i}-Q-h\right)^{2}+2 q_{i}\left(q_{i}-Q+h\right)\right]}{4 h}+w q_{i}-\left(c q_{i}+e q_{i} \sum_{j=1}^{n} q_{j}\right) .
\end{aligned}
$$

By analyzing equation (27), we can obtain Proposition 6.

Proposition 6. The optimal solution in an order flow proportion revenue-sharing supply chain when the urban joint distribution company bearing cost risks satisfies the following equation: $\left(q_{i}^{*}, Q^{*}\right)=\arg \max \left\{\pi_{i}^{r f 2}\left(\Lambda_{0}^{r f 2}\right), \pi_{i}^{r f 2}\right.$
$\left.\left(\Lambda_{q_{i}}^{r f 2}, \Lambda_{Q}^{r f 2}\right)\right\}$, where $\Lambda_{0}^{r f 2}$ is the boundary of the feasible region of the decision variable and $\left(\Lambda_{q_{i}}^{r f 2}, \Lambda_{Q}^{r f 2}\right)$ denotes all the maximum points of the decision function in the feasible region of the decision variable. It also satisfies the following system of equations. 


$$
\left\{\begin{array}{l}
\left(-\frac{1}{\alpha+\beta}+\frac{1}{(\alpha+\beta)(n-1(\alpha / \beta))}-\frac{4 h e}{h-Q^{*}}\right) \frac{\left[-q_{i}^{* 2}-\left(q_{i}^{*}-Q^{*}-h\right)^{2}+2 q_{i}^{*}\left(q_{i}^{*}-Q^{*}+h\right)\right]}{4 h} \\
+\left(\frac{\lambda_{i} d+Q^{*}-q_{i}^{*}}{\alpha+\beta}+\frac{\sum_{j=1}^{n} q_{j}^{*}-n Q^{*}-d}{(\alpha+\beta)(n-1(\alpha / \beta))}-\frac{2 h\left(c+2 e \sum_{j=1}^{n} q_{j}^{*}\right)+(k-v) Q^{*}-h(k+v)}{h-Q^{*}}-c_{i}\right) \\
+\frac{2 h\left(c+2 e \sum_{j=1}^{n} q_{j}^{*}\right)+(k-v) Q^{*}-h(k+v)}{h-Q^{*}}+\frac{4 h e}{h-Q^{*}} q_{i}^{*}-\left(c+e q_{i}^{*}+e \sum_{j=1}^{n} q_{j}^{*}\right)=0, \\
\Lambda_{q_{i}}^{r f 2}=\left\{q_{i}^{*}\right\}, q_{i}^{*} \in \Lambda_{q_{i}}^{r f 2} ; \Lambda_{Q}^{r f 2}=\left\{Q^{*}\right\}, Q^{*} \in[-h, h] .
\end{array}\right.
$$

Proof. Refer to the Appendix.

Similar to the centralized and decentralized optimal solution, the implicit expression can be derived through Proposition 6. We could see that the dimensions of the decision-making variables in Proposition 6 are similar to that in Proposition 3 because the urban joint distribution company bears cost risk in both the situations. After giving other corresponding parameters, we could simulate and present the optimal decision through numerical examples.
5.2.2. Revenue-Sharing under Factor Combination When the Urban Joint Distribution Company Bears Cost Risk. The shared factor combination coefficient for logistics company $i$ is as follows:

$$
\begin{aligned}
& \left(\gamma_{1}, \gamma_{2}, \gamma_{3}, \ldots, \gamma_{n}\right), \quad \forall \gamma_{i} \in[0,1], \\
& \sum_{i=1}^{n} \gamma_{i}=1
\end{aligned}
$$

where $\pi_{i}^{r r 2}$ can be obtained as follows, and the detail of the following equation is shown in Appendix (A.9):

$$
\begin{aligned}
\pi_{i}^{r r 2}= & \left(\frac{\lambda_{i} d+Q-q_{i}}{\alpha+\beta}+\frac{\sum_{j=1}^{n} q_{j}-n Q-d}{(\alpha+\beta)(n-1-(\alpha / \beta))}-\frac{2 h\left(c+2 e \sum_{j=1}^{n} q_{j}\right)+(k-v) Q-h(k+v)}{h-Q}-c_{i}\right) \\
& \frac{\left[-q_{i}^{2}-\left(q_{i}-Q-h\right)^{2}+2 q_{i}\left(q_{i}-Q+h\right)\right]}{4 h}+\gamma_{i}\left\{w \sum_{i=1}^{n} q_{j}-\left[c \sum_{i=1}^{n} q_{j}+e\left(\sum_{i=1}^{n} q_{i}\right)^{2}\right]\right\} .
\end{aligned}
$$

By analyzing equation (30), we can obtain Proposition 7.

Proposition 7. The optimal solution in a factor combination revenue-sharing supply chain when the urban joint distribution company bearing cost risks satisfies the following equation: $\quad\left(q_{i}^{*}, Q^{*}\right)=\arg \max \left\{\pi_{i}^{r r 2}\left(\Lambda_{0}^{r r 2}\right), \pi_{i}^{r r 2}\left(\Lambda_{q_{i}}^{r r 2}, \Lambda_{Q}^{r r 2}\right)\right\}$, where $\Lambda_{0}^{r r 2}$ is the boundary of the feasible region of the decision variable and $\left(\Lambda_{q_{i}}^{r r 2}, \Lambda_{Q}^{r r 2}\right)$ denotes all the maximum points of the decision function in the feasible region of the decision variable. It also satisfies the following system of equations:

$$
\left\{\begin{array}{l}
\left(-\frac{1}{\alpha+\beta}+\frac{1}{(\alpha+\beta)(n-1-(\alpha / \beta))}-\frac{4 h e}{h-Q^{*}}\right) \frac{\left[-q_{i}^{* 2}-\left(q_{i}^{*}-Q^{*}-h\right)^{2}+2 q_{i}^{*}\left(q_{i}^{*}-Q^{*}+h\right)\right]}{4 h} \\
+\left(\frac{\lambda_{i} d+Q^{*}-q_{i}^{*}}{\alpha+\beta}+\frac{\sum_{j=1}^{n} q_{j}^{*}-n Q^{*}-d}{(\alpha+\beta)(n-1-(\alpha / \beta))}-\frac{2 h\left(c+2 e \sum_{j=1}^{n} q_{j}^{*}\right)+(k-v) Q^{*}-h(k+v)}{h-Q^{*}}-c_{i}\right) \\
+\gamma_{i} \frac{2 h\left(c+2 e \sum_{j=1}^{n} q_{j}^{*}\right)+(k-v) Q^{*}-h(k+v)}{h-Q^{*}}+\gamma_{i} \frac{4 h e}{h-Q^{*}} \sum_{i=1}^{n} q_{i}^{*}-\gamma_{i}\left(c+2 e \sum_{j=1}^{n} q_{j}^{*}\right)=0, \\
\Lambda_{q_{i}}^{r r 2}=\left\{q_{i}^{*}\right\}, q_{i}^{*} \in \Lambda_{q_{i}}^{r r 2} ; \Lambda_{Q}^{r r 2}=\left\{Q^{*}\right\}, Q^{*} \in[-h, h] .
\end{array}\right.
$$


Proof. Refer to the Appendix.

Similar to the centralized and decentralized optimal solution, the implicit expression can be derived through Proposition 7. We could will simulate and present the optimal decision through numerical examples after giving other corresponding parameters.

\section{Cost-Sharing Cooperative Decision}

In this section, we will further study the cooperation mechanism problem of the sharing logistics distribution service mode with the risk cost-sharing contracts, which mainly focuses on the analysis of the following three different risk cost-sharing strategies:

(1) Each logistics enterprise shares the risk cost caused by insufficient logistics service supply and excess logistics service supply according to the proportion of order flow (see Section 6.1).

(2) The urban joint distribution company and each logistics company bear the risk cost of insufficient and excess logistics service supply according to the single factor combination $\left(\phi_{0}, \phi_{1}, \phi_{2}, \phi_{3}, \ldots, \phi_{n}\right)$, where $\phi_{0}=1-\sum_{i=1}^{i=n} \phi_{i}, \forall \phi_{i} \in[0,1], i=0,1,2,3, \ldots, n$ (see Section 6.2).
(3) The urban joint distribution company and each logistics company shall bear the costs caused by the insufficient supply of logistics services and the oversupply according to the multifactor combination $\left(\theta_{0}, \theta_{1}, \theta_{2}, \theta_{3}, \ldots, \theta_{n}\right)\left(\gamma_{0}, \gamma_{1}, \gamma_{2}, \gamma_{3}, \ldots, \gamma_{n}\right)$, where $\theta_{0}=1-\sum_{i=1}^{i=n} \theta_{i}, \gamma_{0}=1-\sum_{i=1}^{i=n} \gamma_{i}, \forall \phi_{i}, \gamma_{i} \in[0,1], i=$ $0,1,2,3, \ldots, n$ (see Section 6.3).

6.1. Order Flow Proportion Cost-Sharing Strategy. Each logistics company shares the risk cost due to insufficient and excessive supply of logistics services according to the proportion of order flow contribution, which is as follows:

$$
\left(\frac{q_{1}}{\sum_{j=1}^{n} q_{j}}, \frac{q_{2}}{\sum_{j=1}^{n} q_{j}}, \frac{q_{3}}{\sum_{j=1}^{n} q_{j}}, \ldots, \frac{q_{n}}{\sum_{j=1}^{n} q_{j}}\right) .
$$

The optimal decision of the urban joint distribution company is given by the following equation:

$$
w=c+2 e \sum_{j=1}^{n} q_{j}
$$

where $\pi_{i}^{r s 1}$ can be obtained as follows, and the detail of the following equation is shown in Appendix (A.10):

$$
\begin{aligned}
\pi_{i}^{r s 1}= & \frac{\left(p_{i}-c-2 e \sum_{j=1}^{n} q_{j}-c_{i}\right)}{4 h}\left[q_{i}^{2}-\left(\widehat{x}_{i}-h\right)^{2}\right]+\frac{q_{i}\left(p_{i}-c-2 e \sum_{j=1}^{n} q_{j}-c_{i}\right)}{2 h}\left(\widehat{x}_{i}+h-q_{i}\right) \\
& -\frac{q_{i}}{\sum_{j=1}^{n} q_{j}} \sum_{j=1}^{n}\left\{\frac{\left(c+2 e \sum_{j=1}^{n} q_{j}-v+k\right)}{4 h} q_{j}^{2}-q_{j}\left[\frac{\left(c+2 e \sum_{j=1}^{n} q_{j}-v\right)\left(\widehat{x}_{j}-h\right)}{2 h}+\frac{k\left(\hat{x}_{j}+h\right)}{2 h}\right]\right. \\
& \left.+\frac{\left(c+2 e \sum_{j=1}^{n} q_{j}-v\right)}{4 h}\left(\hat{x}_{j}-h\right)^{2}+\frac{k}{4 h}\left(\widehat{x}_{j}+h\right)^{2}\right\} .
\end{aligned}
$$

By analyzing equation (34), we can obtain Proposition 8.

Proposition 8. The optimal solution under order flow proportion cost-sharing strategy satisfies the following equation $\left(p_{i}^{*}, q_{i}^{*}\right)=\arg \max \left\{\pi_{i}^{r s 1}\left(\Lambda_{0}^{r s 1}\right), \pi_{i}^{r s 1}\left(\Lambda_{p_{i}}^{r s 1}, \Lambda_{q_{i}}^{r s 1}\right)\right\}$, where $\Lambda_{0}^{r s 1}$ is the boundary of the feasible region of the decision variable and $\left(\Lambda_{p_{i}}^{r s 1}, \Lambda_{q_{i}}^{r s 1}\right)$ denotes all the maximum points of the decision function in the feasible region of the decision variable. It also satisfies the following system of equations: 


$$
\begin{aligned}
& \left\{\begin{array}{l}
-\frac{e}{2 h}\left[q_{i}^{* 2}-\left(\hat{x}_{i}^{*}-h\right)^{2}\right]+\frac{\left(p_{i}^{*}-c-2 e \sum_{j=1}^{n} q_{j}^{*}-c_{i}\right)}{2 h}\left(\hat{x}_{i}^{*}-h-q_{i}^{*}\right)-\frac{e q_{i}^{*}}{h}\left(\hat{x}_{i}^{*}-h-q_{i}^{*}\right)-\frac{\sum_{j=1}^{n} q_{j}^{*}-q_{i}^{*}}{\left(\sum_{j=1}^{n} q_{j}^{*}\right)^{2} \times} \\
\sum_{j=1}^{n}\left\{\frac{\left(c+2 e \sum_{j=1}^{n} q_{j}^{*}-v+k\right)}{4 h} q_{j}^{* 2}-q_{j}^{*}\left[\frac{\left(c+2 e \sum_{j=1}^{n} q_{j}^{*}-v\right)\left(\hat{x}_{i}^{*}-h\right)}{2 h}+\frac{k\left(\hat{x}_{i}^{*}+h\right)}{2 h}\right]+\frac{\left(c+2 e \sum_{j=1}^{n} q_{j}^{*}-v\right)}{4 h}\left(\hat{x}_{j}^{*}-h\right)^{2}+\frac{k}{4 h}\left(\hat{x}_{j}^{*}+h\right)^{2}\right\}
\end{array}\right. \\
& -\frac{q_{i}^{*}}{\sum_{j=1}^{n} q_{j}^{*}}\left\{\sum_{j=1}^{n}\left[\frac{e}{2 h} q_{j}^{* 2}-\frac{q_{j}^{*} e\left(\hat{x}_{j}^{*}+h\right)}{h}+\frac{e}{2 h}\left(\hat{x}_{j}^{*}-h\right)^{2}\right]+\frac{c+2 e \sum_{j=1}^{n} q_{j}^{*}-v+k}{2 h} q_{i}^{*}-\frac{\left(c+2 e \sum_{j=1}^{n} q_{j}^{*}-v\right)\left(\hat{x}_{i}^{*}-h\right)}{2 h}-\frac{k\left(\hat{x}_{i}^{*}+h\right)}{2 h}\right\}=0, \\
& \frac{1}{4 h}\left[q_{1}^{* 2}-\left(\hat{x}_{i}^{*}-h\right)^{2}\right]+\frac{\alpha\left(p_{i}^{*}-c-2 e \sum_{j=1}^{n} q_{j}^{*}-c_{i}\right)}{2 h}\left(\hat{x}_{i}^{*}-h-q_{i}^{*}\right)+\frac{q_{i}^{*}}{2 h}\left(\hat{x}_{i}^{*}+h-q_{i}^{*}\right)-\frac{q_{i}^{*}}{\sum_{j=1}^{n} q_{j}^{*} \times} \\
& \left\{\begin{array}{c}
{\left[-\frac{\beta q_{i}^{*}\left(c+2 e \sum_{j=1}^{n} q_{j}^{*}-v\right)}{2 h}-\frac{\beta k q_{j}^{*}}{2 h}+\frac{\beta\left(c+2 e \sum_{j=1}^{n} q_{j}^{*}-v\right)}{2 h}\left(\hat{x}_{j}^{*}-h\right)+\frac{\beta k}{2 h}\left(\hat{x}_{j}^{*}+h\right)\right]} \\
\sum_{\substack{j=1 \\
j \neq 1}}^{n} \frac{\alpha q_{i}^{*}\left(c+2 e \sum_{j=1}^{n} q_{j}^{*}-v\right)}{2 h}+\frac{\alpha k q_{i}^{*}}{2 h}-\frac{\alpha\left(c+2 e \sum_{j=1}^{n} q_{j}^{*}-v\right)}{2 h}\left(\hat{x}_{i}^{*}-h\right)+\frac{\alpha k}{2 h}\left(\hat{x}_{i}^{*}+h\right)
\end{array}\right\}=0, \\
& \Lambda_{p_{i}}^{r s 1}=\left\{p_{i}^{*}\right\}, p_{i}^{*} \in \Lambda_{p_{i}}^{r s 1} ; \Lambda_{q_{i}}^{r s 1}=\left\{q_{i}^{*}\right\}, q_{i}^{*} \in \Lambda_{q_{i}}^{r s 1} .
\end{aligned}
$$

Proof. Refer to the Appendix.

Similar to the centralized and decentralized optimal solution, the implicit expression can be derived through Proposition 8. After giving other corresponding parameters, we could simulate and present the optimal decision through numerical examples.

6.2. Single-Factor Combined Cost-Sharing Strategy. The urban joint distribution company and logistics companies share the risk cost due to insufficient and excessive supply of logistics services according to the unified factor combination coefficient, which is as follows:

$$
\left(\phi_{0}, \phi_{1}, \phi_{2}, \phi_{3}, \ldots, \phi_{n}\right) \text {, where } \phi_{0}=1-\sum_{i=1}^{n} \phi_{i},
$$

$\forall \phi_{i} \in[0,1], i=0,1,2,3, \ldots, n$, where $\pi_{s}^{r s 2}$ can be obtained as follows, and the detail of the following equation is shown in Appendix (A.11):

$$
\begin{aligned}
\pi_{s}^{r s 2}= & w \sum_{i=1}^{n} q_{i}-\left[c \sum_{i=1}^{n} q_{i}+e\left(\sum_{i=1}^{n} q_{i}\right)^{2}\right] \\
& -\phi_{0} \sum_{i=1}^{n}\left\{\frac{(w-v) q_{i}}{2 h}\left(q_{i}-\hat{x}_{i}+h\right)-\frac{(w-v)}{4 h}\right. \\
& \cdot\left[q_{i}^{2}-\left(\hat{x}_{i}-h\right)^{2}\right]+\frac{k}{4 h}\left[\left(\hat{x}_{i}+h\right)^{2}-q_{i}^{2}\right] \\
& \left.-\frac{k q_{i}}{2 h}\left(\hat{x}_{i}+h-q_{i}\right)\right\} .
\end{aligned}
$$

We solve first derivatives of $\pi_{s}^{r s 2}$ with respect to the variable $q_{i}$ and equate it to 0 , thus obtaining the following equation: 


$$
\begin{aligned}
\frac{\partial \pi_{s}^{r s 2}}{\partial q_{i}} & =w-\left(c+2 e \sum_{j=1}^{n} q_{j}\right)-\phi_{0}\left[\frac{(w-v)}{2 h}\left(q_{i}-\hat{x}_{i}+h\right)-\frac{k}{2 h}\left(\hat{x}_{i}+h-q_{i}\right)\right] \\
& =w-\left(c+2 e \sum_{j=1}^{n} q_{j}\right)-\phi_{0}\left[\frac{(w-v+k)}{2 h}\left(q_{i}-\hat{x}_{i}\right)+\frac{w-v-k}{2}\right]=0, \\
w & =\frac{2 h\left(c+2 e \sum_{j=1}^{n} q_{j}\right)+\phi_{0}(k-v)\left(q_{i}-\hat{x}_{i}\right)-\phi_{0} h(k+v)}{\left(2-\phi_{0}\right) h+\phi_{0}\left(\hat{x}_{i}-q_{i}\right)}
\end{aligned}
$$

where $\forall i, j \in N, i \neq j$, and we can obtain $q_{i}-\hat{x}_{i}=q_{j}-\hat{x}_{j}$. Thus, assuming $q_{1}-\hat{x}_{1}=q_{2}-\hat{x}_{2}=q_{3}-\hat{x}_{3}=\cdots=q_{n}-\hat{x}_{n}$ $=Q \in[-h, h]$, then there is $\hat{x}_{i}=q_{i}-Q, i=1,2,3, \ldots, n$. Thus, we obtain the expression of $p_{i}$ as follows:

$$
p_{i}=\frac{\lambda_{i} d+Q-q_{i}}{\alpha+\beta}+\frac{\sum_{i=1}^{n} q_{i}-n Q-d}{(\alpha+\beta)(n-1-(\alpha / \beta))} .
$$

Equations (38) and (39) are the optimal decisions for the urban joint distribution company. Combining equations (38) and (39), we can obtain the profit of the logistics company $i$ as follows and Proposition 9 can be obtained, and the detail of the following equation is shown in Appendix (A.12):

$$
\begin{aligned}
\pi_{i}^{r s 2}= & \frac{\left(p_{i}-w-c_{i}\right)}{4 h}\left[q_{i}^{2}-\left(q_{i}-Q-h\right)^{2}\right]+\frac{q_{i}\left(p_{i}-w-c_{i}\right)}{2 h}(h-Q) \\
& -\phi_{i} \sum_{j=1}^{n}\left\{\frac{(w-v+k)}{4 h} q_{j}^{2}-q_{j}\left[\frac{(w-v)\left(q_{j}-Q-h\right)}{2 h}+\frac{k\left(q_{j}-Q+h\right)}{2 h}\right]+\frac{(w-v)}{4 h}\left(q_{j}-Q-h\right)^{2}+\frac{k}{4 h}\left(q_{j}-Q+h\right)^{2}\right\} .
\end{aligned}
$$

Proposition 9. The optimal solution under single-factor combined cost-sharing strategy satisfies the following equation: $\left(q_{i}^{*}, Q^{*}\right)=\arg \max \left\{\pi_{i}^{r s 2}\left(\Lambda_{0}^{r s 2}\right), \pi_{i}^{r s 2}\left(\Lambda_{q_{i}}^{r s 2}, \Lambda_{Q}^{r s 2}\right)\right\}$, where $\Lambda_{0}^{r s 2}$ is the boundary of the feasible region of the decision variable and $\left(\Lambda_{q_{i}}^{r s 2}, \Lambda_{Q}^{r s 2}\right)$ denotes all the maximum points of the decision function in the feasible region of the decision variable. It also satisfies the following system of equations:

$$
\left\{\begin{array}{l}
\frac{\lambda_{i} d+Q^{*}-q_{i}^{*}}{\alpha+\beta}+\frac{\sum_{j=1}^{n} q_{j}^{*}-n Q^{*}-d}{(\alpha+\beta)(n-1-(\alpha / \beta))}-\frac{2 h\left(c+2 e \sum_{j=1}^{n} q_{j}^{*}\right)+\phi_{0}(k-v) Q^{*}-\phi_{0} h(k+v)}{\left(2-\phi_{0}\right) h-\phi_{0} Q^{*}} \\
-c_{i}+\frac{q_{i}^{* 2}-\left(q_{i}^{*}-Q^{*}-h\right)^{2}+2 q_{i}^{*}\left(h-Q^{*}\right)}{4 h} \\
\times\left[-\frac{1}{\alpha+\beta}+\frac{1}{(\alpha+\beta)(n-1-(\alpha / \beta))}-\frac{4 h e}{\left(2-\phi_{0}\right) h-\phi_{0} Q^{*}}\right]-\phi_{i}\left[\frac{\left(h+Q^{*}\right)^{2}}{4 h} \times \frac{4 n h e}{\left(2-\phi_{0}\right) h-\phi_{0} Q^{*}}+\frac{k}{2 h}+\frac{k\left(2 q_{i}^{*}-Q^{*}+h\right)}{2 h}\right]=0, \\
\Lambda_{q_{i}}^{r s 2}=\left\{q_{i}^{*}\right\}, q_{i}^{*} \in \Lambda_{q_{i}}^{r s 2} ; \Lambda_{Q}^{r s 2}=\left\{Q^{*}\right\}, Q^{*} \in[-h, h] .
\end{array}\right.
$$

Proof. Refer to the Appendix.

Similar to the centralized and decentralized optimal solution, the implicit expression can be derived through
Proposition 9. We could simulate and present the optimal decision through numerical examples after giving other corresponding parameters. 
6.3. Multifactor Combined Cost-Sharing Strategy. They bear the cost incurred due to the insufficient supply of logistics services according to the coefficient $\theta_{i}$ and bear the cost incurred due to the excess supply of logistics services according to the coefficient $\gamma_{i}$, which is as follows:

$$
\begin{aligned}
& \left(\theta_{0}, \theta_{1}, \theta_{2}, \theta_{3}, \ldots, \theta_{n}\right),\left(\omega_{0}, \omega_{1}, \omega_{2}, \omega, \ldots, \omega_{n}\right), \\
& \text { where } \theta_{0}=1-\sum_{i=1}^{n} \theta_{i}, \gamma_{0}=1-\sum_{i=1}^{n} \omega_{i}, \\
& \qquad \forall \phi_{i}, \omega_{i} \in[0,1], i=0,1,2,3, \ldots, n,
\end{aligned}
$$

where $\pi_{s}^{r s 3}$ can be obtained as follows, and the detail of the following equation is shown in Appendix (A.13):

$$
\begin{aligned}
\pi_{s}^{r s 3}= & w \sum_{i=1}^{n} q_{i}-\left[c \sum_{i=1}^{n} q_{i}+e\left(\sum_{i=1}^{i=n} q_{i}\right)^{2}\right]-\theta_{0} \sum_{j=1}^{n}\left\{\frac{(w-v) q_{j}}{2 h}\left(q_{j}-\widehat{x}_{j}+h\right)-\frac{(w-v)}{4 h}\left[q_{j}^{2}-\left(\widehat{x}_{j}-h\right)^{2}\right]\right\} \\
& -\omega_{0} \sum_{j=1}^{n}\left\{\frac{k}{4 h}\left[\left(\widehat{x}_{j}+h\right)^{2}-q_{j}^{2}\right]-\frac{k q_{j}}{2 h}\left(\widehat{x}_{j}+h-q_{j}\right)\right\} .
\end{aligned}
$$

We solve first derivatives of the function $\pi_{s}^{r s 3}$ with respect to the variable $q_{i}$ and equate it to 0 , thus obtaining equation (38):

$$
\begin{aligned}
\frac{\partial \pi_{s}^{r s 3}}{\partial q_{i}} & =w-\left(c+2 e \sum_{j=1}^{n} q_{j}\right)-\theta_{0} \frac{(w-v)\left(q_{i}-\hat{x}_{i}+h\right)}{2 h}-\omega_{0} \frac{k\left(q_{i}-\hat{x}_{i}-h\right)}{2 h}=0, \\
w & =\frac{2 h\left(c+2 e \sum_{j=1}^{n} q_{j}\right)-\theta_{0} v\left(q_{i}-\hat{x}_{i}+h\right)+\omega_{0} k\left(q_{i}-\hat{x}_{i}-h\right)}{\left(2-\theta_{0}\right) h-\theta_{0}\left(q_{i}-\hat{x}_{i}\right)},
\end{aligned}
$$

Where $\forall i, j \in N, i \neq j$, and we can obtain $q_{i}-\widehat{x}_{i}=q_{j}-\widehat{x}_{j}$. Thus, assuming $q_{1}-\widehat{x}_{1}=q_{2}-\widehat{x}_{2}=q_{3}-\widehat{x}_{3}=\cdots=q_{n}-\widehat{x}_{n}$ $=Q \in[-h, h]$, then there is $\widehat{x}_{i}=q_{i}-Q, i=1,2,3, \ldots, n$, obtaining the expression of $p_{i}$ as equation (39):

$$
p_{i}=\frac{\lambda_{i} d+Q-q_{i}}{\alpha+\beta}+\frac{\sum_{i=1}^{n} q_{i}-n Q-d}{(\alpha+\beta)(n-1-(\alpha / \beta))} \text {. }
$$

Equations (44) and (45) are the optimal decisions for the urban joint distribution company. Combining equations (44) and (45), we can obtain the profit of the logistics company $i$ as follows and derive Proposition 10, and the detail of equation (46) is shown in Appendix (A.14):

$$
\begin{aligned}
\pi_{i}^{r s 3}= & \frac{\left(p_{i}-w-c_{i}\right)}{4 h}\left[q_{i}^{2}-\left(q_{i}-Q-h\right)^{2}\right]+\frac{q_{i}\left(p_{i}-w-c_{i}\right)}{2 h}(h-Q) \\
& -\theta_{i} \sum_{j=1}^{n}\left\{\frac{(w-v) q_{j}}{2 h}(Q+h)-\frac{(w-v)}{4 h}\left[q_{j}^{2}-\left(q_{j}-Q-h\right)^{2}\right]\right\} \\
& -\omega_{i} \sum_{j=1}^{n}\left\{\frac{k}{4 h}\left[\left(q_{j}-Q+h\right)^{2}-q_{j}^{2}\right]-\frac{k q_{j}}{2 h}(h-Q)\right\} .
\end{aligned}
$$


Proposition 10. The optimal solution under multifactor combined cost-sharing strategy satisfies the following equation: $q i *, Q *=\operatorname{argmax} \pi i r s 3 \Lambda 0 r s 3$, $\pi i r s 3 \Lambda$ qirs3, $1 Q$ rs3, where $\Lambda$ Ors 3 is the boundary of the feasible region of the decision variable and $\Lambda$ qirs $3, \Lambda$ Qrs 3 denotes all the maximum points of the decision function in the feasible region of the decision variable. It also satisfies the following system of equations:

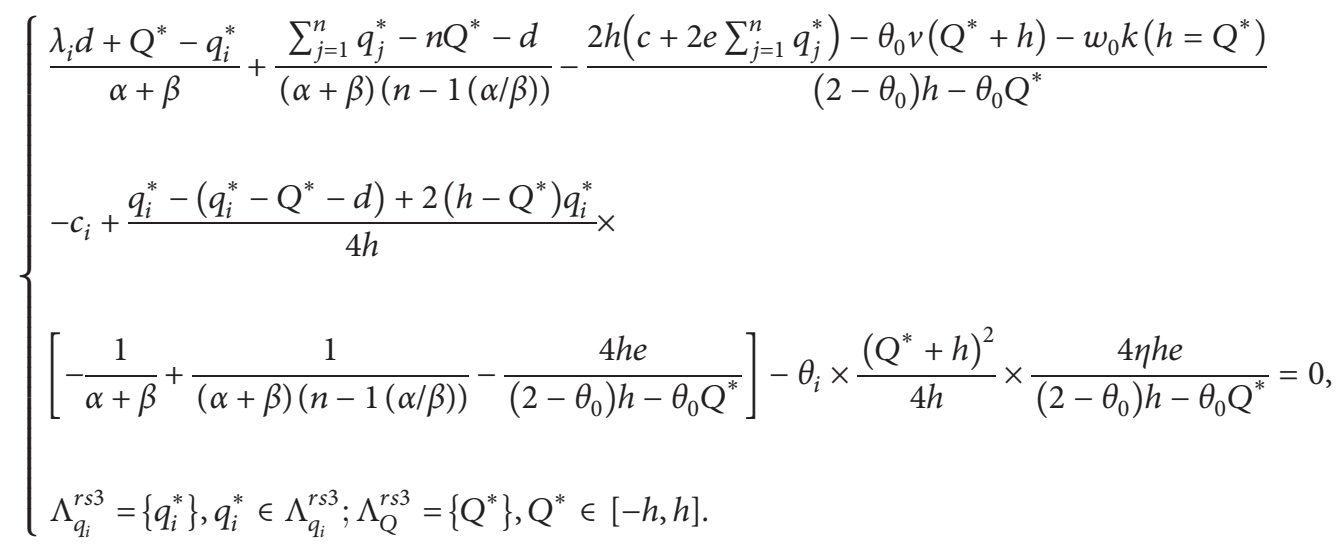

Proof. Refer to the Appendix.

Similar to the centralized and decentralized optimal solution, the implicit expression can be derived through Proposition 10. After giving other corresponding parameters, we could simulate and present the optimal decision through numerical examples.

\section{Case Analysis}

7.1. Case 1: Provincial Joint Distribution. We obtained relevant data for the two-echelon logistics service supply chain composed of Tongyue, Yusheng, Xinye, Wangyong, and Yuanyao in Sichuan Province for October, 2019, through interviews and surveys. Tongyue, Yusheng, and Xinye are selected for the analysis, and the model parameters are shown in Table 1.

The change trend of the total profit of the provincial supply chain under different cooperation model decisions is shown in Table 2 and Figure 1. We obtain some conclusions from Table 2. The total profit of the decentralized supply chain when the urban joint distribution company bears the cost risk is higher than that of the decentralized supply chain when the logistics company bears the cost risk. When the logistics companies bear the cost risks, the order flow proportion and the factor combination revenue-sharing strategy cannot achieve Pareto improvement of the total profit of the supply chain, where their values are equal to the total profit of the decentralized supply chain when the logistics company bears the cost risk. When the urban joint distribution company bears cost risks, the order flow proportion and the factor combination revenue-sharing strategy can achieve Pareto improvement of the total profit of the supply chain and the order flow proportion revenue-sharing strategy is better than factor combination revenue-sharing strategy. Although the Pareto improvement of the total profit of the supply chain failed to achieve supply chain coordination, compared to the total profit of the decentralized supply chain, the profit of the supply chain in the order flow proportion revenue-sharing supply chain when urban joint distribution company bears cost risks increased by $55.48 \%$.

As shown in Figure 1, under most factor combinations, the total profit of the supply chain under the single-factor combination cost-sharing strategy is significantly higher than that under the two-factor combination cost-sharing strategy. At the same time, the total profit of the supply chain under the cost-sharing strategy of logistics company order flow proportion is higher than that under the single factor combination-sharing strategy in the vast majority of factor combinations. However, there are certain factor combinations that make the total supply chain profit under the single-factor combined cost-sharing strategy higher than that under the logistics company's order flow proportion cost-sharing strategy. The total profit of the supply chain under the cost-sharing strategy of logistics company order flow proportion is higher than that under the two-factor combined cost-sharing strategy. Moreover, the total profit of the supply chain under the cost-sharing strategy is higher than that under the revenue-sharing strategy in most factor combinations. In particular, the order flow proportion costsharing strategy is better than the revenue-sharing strategy.

7.2. Case 2: Interprovincial Joint Distribution. The interprovincial joint distribution case uses data from the annual financial reports of the listed express companies A, B, C, and D for October, 2018, as shown in Tables 3 and 4.

The market share and market scale of various express delivery companies are shown in Table 3 . The market shares are $19.63 \%, 25.43 \%, 23.67 \%$, and $31.26 \%$ for the listed express companies $\mathrm{A}, \mathrm{B}, \mathrm{C}$, and $\mathrm{D}$, respectively.

Referring to Wei et al. [23]; we estimate $\alpha$ and $\beta$ by the least square method. As shown in Table 4, the sensitivity coefficients of the market demand of each express company 
TABLE 1: Model parameters.

\begin{tabular}{lcccccc}
\hline$\alpha$ & $\beta$ & $\lambda_{1}$ & $\lambda_{2}$ & $\lambda_{3}$ & $d$ & $c$ \\
12 & 2 & 0.26 & 0.63 & 0.11 & 120 \\
\hline$c_{1}$ & $c_{2}$ & $c_{3}$ & $v$ & $k$ & $h$ & 2 \\
1 & 0.8 & 1.5 & 5 & 6 & 6 & 0.01 \\
\hline
\end{tabular}

TABLE 2: Total profit of the provincial supply chain under different cooperative decision-making situations $(\times 103)$.

\begin{tabular}{lllllllll}
\hline$\pi_{\mathrm{sc}}^{c}$ & $\pi_{\mathrm{sc}}^{d 1}$ & $\pi_{\mathrm{sc}}^{d 2}$ & $\pi_{\mathrm{sc}}^{r f 1}$ & $\pi_{\mathrm{sc}}^{r f 2}$ & $\pi_{\mathrm{sc}}^{r r 1}$ & $\pi_{\mathrm{sc}}^{r r 2}$ & $\pi_{\mathrm{sc}}^{r s 1}$ & $\pi_{\mathrm{sc}}^{r s 2}$ \\
\hline 21.1 & 1.94 & 5.84 & 1.94 & 9.08 & 1.94 & 5.76 & 1.58 & 5.84 \\
21.1 & 1.94 & 5.84 & 1.94 & 9.08 & 1.94 & 7.23 & 1.58 & 1.00 \\
21.1 & 1.94 & 5.84 & 1.94 & 9.08 & 1.94 & 8.29 & 1.58 & 1.18 \\
21.1 & 1.94 & 5.84 & 1.94 & 9.08 & 1.94 & 7.87 & 1.58 & 1.18 \\
21.1 & 1.94 & 5.84 & 1.94 & 9.08 & 1.94 & 6.83 & 1.58 & 1.76 \\
21.1 & 1.94 & 5.84 & 1.94 & 9.08 & 1.94 & 7.49 & 1.58 & 1.84 \\
21.1 & 1.94 & 5.84 & 1.94 & 9.08 & 1.94 & 8.06 & 1.58 & 1.14 \\
\hline
\end{tabular}

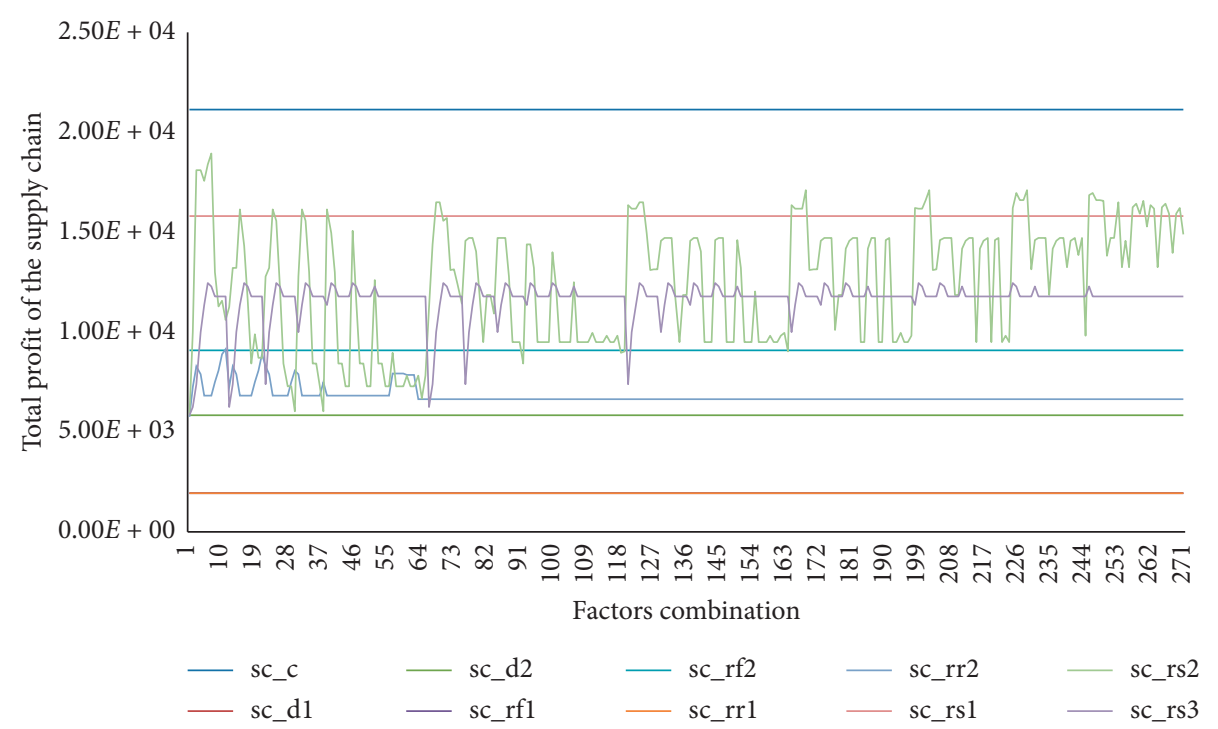

Figure 1: Total profit of the provincial supply chain under the benchmark situation and two different cooperative decision-making situations in case 1 . Note: the abscissa in Figure 1 is the factor combination for revenue-sharing or cost-sharing. To facilitate an intuitive comparison, we have summarized the profit results in all relevant situations into a single graph. However, due to the different factor combinations of revenue-sharing or cost-sharing in different situations, some are single factors, while others are double factors, and the factor combinations are multidimensional. Hence, the abscissa only represents the optimal case of all factor combinations, not a specific parameter value. The abscissa in Figure 2 is the same as that in Figure 1.

TABLE 3: Market size and market share of listed express companies.

\begin{tabular}{lcccc}
\hline$d$ & $\lambda_{1}$ & $\lambda_{2}$ & $\lambda_{3}$ & $\lambda_{4}$ \\
\hline 19954817621 & 0.1971 & 0.2633 & 0.2406 & 0.3209 \\
\hline & $\lambda_{1} d$ & $\lambda_{2} d$ & $\lambda_{3} d$ & $\lambda_{4} d$ \\
& 3933094553 & 5254103480 & 4801129120 & 6403500975 \\
\hline
\end{tabular}

TABLE 4: Estimated parameters of $\alpha$ and $\beta$.

\begin{tabular}{lc}
\hline$\widehat{\alpha}$ & $\widehat{\beta}$ \\
\hline 1342985031.67 & 7601243.14 \\
\hline
\end{tabular}

to its own express service price and the service prices of other express companies are 1342985031.67 and 7601243.14, respectively.

As shown in Table 5, the unit distribution cost of the shared logistics distribution company industry is 1.55 yuan/
TABLE 5: Cost structure information.

\begin{tabular}{lcccc}
\hline$c$ & $c_{1}$ & $c_{2}$ & $c_{3}$ & $c_{4}$ \\
\hline 1.55 & 1.03 & 1.24 & 1.08 & 0.95 \\
\hline
\end{tabular}

piece and the unit transportation cost of each express delivery company, namely, the intercity logistics transportation cost, is $1.03,1.24,1.08$, and 0.95 , respectively.

As shown in Table 6 , the value range of the nonscale economic cost coefficient $e$ for shared logistics distribution companies to provide shared logistics distribution services is set between 0.01 and 0.11 yuan, the residual value of the excess supply of express services $v$ is 1.20 yuan/piece, and the supply of express services is insufficient. The unit opportunity cost $k$ is 2 yuan/piece. At the same time, the characteristic variable of uncertain itemsh is $200,000,000$, which is about $4.44 \%$ of the average market size of several listed express companies. 
TABLE 6: Risk costs.

\begin{tabular}{lccc}
\hline$e$ & $v$ & $k$ & $h$ \\
\hline $0.01 \longrightarrow 0.11$ & 1.25 & 2.03 & 200000000 \\
\hline
\end{tabular}

TABLe 7: Total profit of the interprovincial supply chain under different cooperative decision-making situations $(\times 1016)$.

\begin{tabular}{lllllllll}
\hline$\pi_{\mathrm{sc}}^{c}$ & $\pi_{\mathrm{sc}}^{d 1}$ & $\pi_{\mathrm{sc}}^{d 2}$ & $\pi_{\mathrm{sc}}^{r f 1}$ & $\pi_{\mathrm{sc}}^{r f 2}$ & $\pi_{\mathrm{sc}}^{r r 1}$ & $\pi_{\mathrm{sc}}^{r r 2}$ & $\pi_{\mathrm{sc}}^{r s 1}$ & $\pi_{\mathrm{sc}}^{r s 2}$ \\
\hline 975 & 2.74 & 93.6 & 2.74 & 140 & 2.74 & 296 & 86.6 & 93.6 \\
975 & 2.74 & 93.6 & 2.74 & 140 & 2.74 & 296 & 86.6 & 93.6 \\
975 & 2.74 & 93.6 & 2.74 & 140 & 2.74 & 296 & 86.6 & 199 \\
975 & 2.74 & 93.6 & 2.74 & 140 & 2.74 & 296 & 86.6 & 93.6 \\
975 & 2.74 & 93.6 & 2.74 & 140 & 2.74 & 296 & 86.6 & 93.6 \\
975 & 2.74 & 93.6 & 2.74 & 140 & 2.74 & 296 & 86.6 & 199 \\
975 & 2.74 & 93.6 & 2.74 & 140 & 2.74 & 296 & 86.6 & 93.6 \\
\hline
\end{tabular}

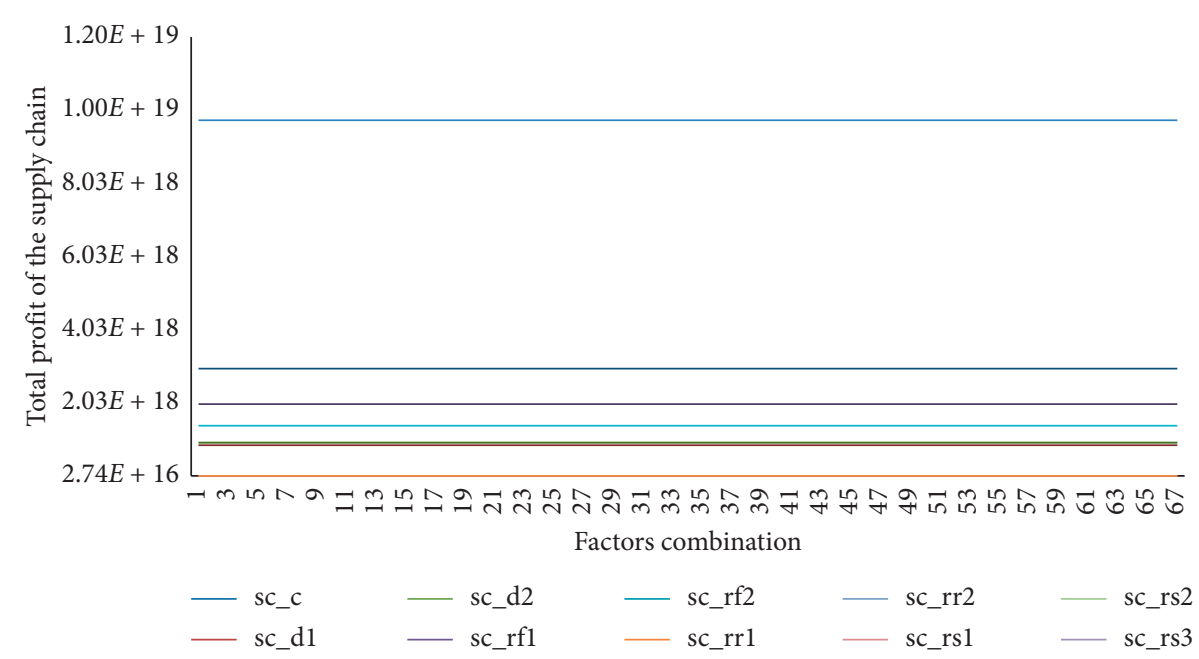

FIGURE 2: Total profit of the interprovincial supply chain under the benchmark situation and two different cooperative decision-making situations in case 2 .

Next, we analyze the change in trend of the total profit of the supply chain under several supply chain decision situations when the combination sharing factor changes, taking centralized and decentralized supply chains as benchmarks. We randomly selected three from the four listed express companies in China for sharing logistics and distribution cooperation to graphically display the effect of the shared cooperation mechanism.

The change in trend of the total profit of the interprovincial supply chain under different cooperation model decisions is shown in Table 7 and Figure 2. We obtain the following conclusions. Under revenue-sharing or cost-sharing cooperation mechanism, not all cooperation scenarios under consideration can achieve Pareto improvement of the total profit of the supply chain, but at least one situation can achieve Pareto improvement. The total profit of the supply chain in an order flow proportion revenue-sharing supply chain and a factor combination revenuesharing supply chain when urban codistribution company bears cost risks and under two-factor combined cost-sharing strategy are higher than that under the decentralized supply chain. The optimal decision of the logistics service supply chain is to choose a factor-combined revenue-sharing cooperation strategy when the city codistribution company bears the risk cost. The total profit of the supply chain has increased by $216.24 \%$ compared to the total profit of the decentralized supply chain.

\section{Conclusions}

We design a two-echelon logistics service supply chain composed of an urban joint distribution company and $N$ logistics companies. The urban joint distribution company is jointly established by $N$ logistics companies that cooperate through equity investment. The equity investment ratio is uniquely determined by the revenue-sharing or cost-sharing factor that achieves Pareto improvement of the total profit of the supply chain. Then, based on the centralized and decentralized service supply chain, four different types of cooperation strategies are comparatively analyzed under the revenue-sharing mechanism, and three different types of cooperation strategies are analyzed under the cost-sharing mechanism. As the solution of optimal decision-making involves binary cubic equations, only implicit expressions could be obtained. Similar to Zhang et al. [22], we use some algorithms to solve the optimal decision in the case analysis. 
Both case 1 and case 2 show that whether to choose revenue-sharing or cost-sharing mechanism, not all cooperation scenarios considered in the study can achieve Pareto improvement of the total profit of the supply chain, but at least one situation can achieve Pareto improvement; thus, cooperation is the better choice.

The optimal decision of the logistics service supply chain in case 1 is to choose the logistics company's order flow proportion cost-sharing cooperation strategy. The equity investment proportion of the logistics company $i$ is $\left(q_{i} / \sum_{j=1}^{n} q_{j}\right)$. This is contrary to the conclusion of Yang and Chen [31] and $\mathrm{Yu}$ et al. [33] who find that revenue-sharing contracts are preferred for the supply chain. A possible reason is that the two sides of the supply chain are independent in their studies, which is not the case in our study. The joint distribution company is jointly established by the logistics companies through equity cooperation. As it is related to revenue-sharing, cost-sharing is much more important.

The optimal decision of the logistics service supply chain in case 2 is to choose a factor combination revenue-sharing cooperation strategy when the urban joint distribution company bears the risk cost. The equity investment proportion of the logistics company $i$ is $\gamma_{i}$. This is consistent with the research conclusions of Yang and Chen [31] and Yu et al. [33]; that is, revenue-sharing contracts are preferred for the supply chain. Even when revenue-sharing and costsharing are both optional, cost-sharing is dispensable.

The findings of the study have some implications for managements. First, the sharing economy model is promising in the field of urban distribution and cost reduction and efficiency increase could be achieved through building a common distribution platform. Second, different logistics companies can learn from the equity investment cooperation methods mentioned in this study when deciding which way to cooperate to build a joint distribution platform. The joint distribution company is jointly established by the logistics companies through equity cooperation, which is related to both the revenue-sharing and cost-sharing contracts. We propose an actual strategy that guides the logistics company scientifically steps into the cooperation based on both the supply chain coordination theory and the corporate governance (equity investment) theory. Third, our research conclusions have pointed out a stable vertical integration path for the scientific development of small and medium logistics companies, and this path can avoid the detours that many enterprises have taken on the road of integration to a certain extent.

There are several limitations worth further exploration. First, this study does not consider the issue of logistics service supply chain coordination. Second, it does not consider the risk preference of logistics service supply chain members.

Some future research directions are as follows. First, the issue of equity cooperation mechanism in the sharing logistics service supply chain can be considered when the service price changes with the distribution product, region, distance, and time. Second, the sharing logistics service supply chain coordination can be considered in future research. Third, the different risk preferences of logistics service supply chain members can be discussed in the future study on the design of equity cooperation mechanism in the sharing logistics service supply chain.

\section{Notations}

\begin{tabular}{|c|c|}
\hline$x_{i}:$ & Demand function of logistics company $i$ \\
\hline$\hat{x}_{i}:$ & Mean of $x_{i}$ \\
\hline$f\left(x_{i}\right):$ & Probability density function of $x_{i}$ \\
\hline$F\left(x_{i}\right):$ & Probability distribution function of $x_{i}$ \\
\hline$\varepsilon:$ & Error of $x_{i}, \varepsilon \sim U[-h, h]$ \\
\hline$h:$ & $\begin{array}{l}\text { The interval value of the uniform distribution } \\
\varepsilon \sim U[-h, h]\end{array}$ \\
\hline$d:$ & $\begin{array}{l}\text { Market size of the entire subdivision } \\
\text { distribution field }\end{array}$ \\
\hline$e:$ & Unit nonscale economic cost \\
\hline$p_{i}:$ & Unit service price of logistics company $i$ \\
\hline$q_{i}:$ & Service order quantity of logistics company $i$ \\
\hline$c:$ & $\begin{array}{l}\text { Unit service cost of the urban joint distribution } \\
\text { company }\end{array}$ \\
\hline$c_{i}:$ & Unit service cost of logistics company $i$ \\
\hline$\alpha:$ & $\begin{array}{l}\text { The sensitivity coefficient of the demand of } \\
\text { logistics company } i \text { to its unit service price }\end{array}$ \\
\hline$\beta:$ & $\begin{array}{l}\text { The sensitivity coefficient of the demand of } \\
\text { logistics company } i \text { to the unit service price of } \\
\text { other logistics companies }\end{array}$ \\
\hline$\lambda_{i}:$ & Market share of the logistics company $i$ \\
\hline$w:$ & Unit price of the urban joint distribution service \\
\hline$v:$ & $\begin{array}{l}\text { Unit residual value of the urban joint } \\
\text { distribution company }\end{array}$ \\
\hline$k$ : & $\begin{array}{l}\text { Unit opportunity cost of the urban joint } \\
\text { distribution company }\end{array}$ \\
\hline$\gamma_{i}:$ & $\begin{array}{l}\text { Shared factor combination coefficient for } \\
\text { logistics company } i\end{array}$ \\
\hline
\end{tabular}

$\phi_{i}$ : $\quad$ Unified factor combination coefficient of the urban joint distribution company and logistics companies that share the risk cost due to insufficient and excessive supply of logistics services

$\theta_{i}$ : $\quad$ Coefficient of the urban joint distribution company and logistics companies that bear the cost incurred due to the insufficient supply of logistics services

$\omega_{i}$ : Coefficient of the urban joint distribution company and logistics companies that bear the cost incurred due to the excess supply of logistics services

$\pi_{s}: \quad$ Profit function of the urban joint distribution company

$\pi_{i}$ : $\quad$ Profit function of logistics company $i$

$O_{i}(\vec{p}, \vec{q})$ : Overcapacity cost

$U_{i}(\vec{p}, \vec{q})$ : Undercapacity cost

$\pi_{\mathrm{sc}}^{c}$ : $\quad$ Profit of the centralized supply chain under joint distribution cooperation

$\pi_{s}^{d 1}$ : $\quad$ Profit of the urban joint distribution company in a decentralized supply chain when logistics companies bear cost risks

$\pi_{i}^{d 1}: \quad \quad \quad \quad$ Profit of logistics company $i$ in a decentralized supply chain when logistics companies bear cost risks

$\pi_{\mathrm{sc}}^{d 1}: \quad \pi_{\mathrm{sc}}^{d 1}=\pi_{s}^{d 1}+\sum \pi_{i}^{d 1}$

$\pi_{s}^{d 2}$ : $\quad$ Profit of the urban joint distribution company in a decentralized supply chain when the urban joint distribution company bears cost risks 
$\pi_{i}^{d 2}: \quad \quad \quad$ Profit of logistics company $i$ in a decentralized supply chain when the urban joint distribution company bears cost risks

$\pi_{\mathrm{sc}}^{d 2}: \quad \pi_{\mathrm{sc}}^{d 2}=\pi_{s}^{d 2}+\sum \pi_{i}^{d 2}$

$\pi_{s}^{r 1}$ : $\quad$ Profit of the urban joint distribution company in a revenue-sharing supply chain when logistics companies bear cost risks

$\pi_{i}^{r f 1}: \quad$ Profit of logistics company $i$ in an order flow proportion revenue-sharing supply chain when logistics companies bear cost risks

$\pi_{\mathrm{sc}}^{r f 1}: \quad \pi_{\mathrm{sc}}^{r f 1}=\pi_{s}^{r 1}+\sum \pi_{i}^{r f 1}$

$\pi_{i}^{r r 1}$ : $\quad$ Profit of logistics company $i$ in a factor combination revenue-sharing supply chain when logistics companies bear cost risks

$\pi_{\mathrm{sc}}^{r r 1}: \quad \pi_{\mathrm{sc}}^{r r 1}=\pi_{s}^{r 1}+\sum \pi_{i}^{r r 1}$

$\pi_{s}^{r 2}$ : $\quad$ Profit of the urban joint distribution company in a revenue-sharing supply chain when the urban joint distribution company bears cost risks

$\pi_{i}^{r f 2}:$

$\pi_{\mathrm{sc}}^{r f 2}: \quad \pi_{\mathrm{sc}}^{r f 2}=\pi_{s}^{r 2}+\sum \pi_{i}^{r f 2}$

$\pi_{i}^{r r 2}$ : $\quad$ Profit of logistics company $i$ in a factor combination revenue-sharing supply chain when the urban joint distribution company bears cost risks

$\pi_{\mathrm{sc}}^{r r 2}: \quad \pi_{\mathrm{sc}}^{r r 2}=\pi_{s}^{r 2}+\sum \pi_{i}^{r r 2}$

$\pi_{s}^{r s 1}$ : $\quad$ Profit of the urban joint distribution company under order flow proportion cost-sharing strategy

$\pi_{i}^{r s 1}$ : $\quad$ Profit of logistics company $i$ under order flow proportion cost-sharing strategy

$\pi_{\mathrm{sc}}^{r s 1}: \quad \pi_{\mathrm{sc}}^{r s 1}=\pi_{s}^{r s 1}+\sum \pi_{i}^{r s 1}$

$\pi_{s}^{r s 2}$ : $\quad$ Profit of the urban joint distribution company under single factor combined cost-sharing strategy

$\pi_{i}^{r s 2}$ : $\quad$ Profit of logistics company $i$ under single factor combined cost-sharing strategy

$\pi_{\mathrm{sc}}^{r s 2}: \quad \pi_{\mathrm{sc}}^{r s 2}=\pi_{s}^{r s 2}+\sum \pi_{i}^{r s 2}$

$\pi_{s}^{r s 3}$ : Profit of the urban joint distribution company under two-factor combined cost-sharing strategy

$\pi_{i}^{r s 3}$ : $\quad$ Profit of logistics company $i$ under two-factor combined cost-sharing strategy

$\pi_{\mathrm{sc}}^{r s 3}: \quad \pi_{\mathrm{sc}}^{r s 3}=\pi_{s}^{r s 3}+\sum \pi_{i}^{r s 3}$.

\section{Appendix}

The detail of equation (6) is as follows:

$$
\begin{aligned}
\pi_{\mathrm{sc}}^{c}= & \pi_{s}+\sum_{i=1}^{n} \pi_{i}-\sum_{i=1}^{n} O_{i}(\vec{p}, \vec{q})-\sum_{i=1}^{n} U_{i}(\vec{p}, \vec{q}) \\
= & w \sum_{i=1}^{n} q_{i}-\left[c \sum_{i=1}^{n} q_{i}+e\left(\sum_{i=1}^{n} q_{i}\right)^{2}\right] \\
& +\sum_{i=1}^{n}\left[\int_{\hat{x}_{i}-h}^{q_{i}}\left(p_{i}-w-c_{i}\right) x_{i} f\left(x_{i}\right) \mathrm{d} x_{i}+\int_{q_{i}}^{\hat{x}_{i}+h}\left(p_{i}-w-c_{i}\right) q_{i} f\left(x_{i}\right) \mathrm{d} x_{i}-\int_{\hat{x}_{i}-h}^{q_{i}}(w-v)\left(q_{i}-x_{i}\right) f\left(x_{i}\right) \mathrm{d} x_{i}-\int_{q_{i}}^{\hat{x}_{i}+h} k\left(x_{i}-q_{i}\right) f\left(x_{i}\right) \mathrm{d} x_{i}\right] \\
= & \sum_{i=1}^{n}\left[\int_{\hat{x}_{i}-h}^{q_{i}}\left(p_{i}-c_{i}-v\right) x_{i} f\left(x_{i}\right) \mathrm{d} x_{i}+v q_{i} \int_{\hat{x}_{i}-h}^{q_{i}} f\left(x_{i}\right) \mathrm{d} x_{i}+\int_{q_{i}}^{\hat{x}_{i}+h}\left(p_{i}-c_{i}+k\right) q_{i} f\left(x_{i}\right) \mathrm{d} x_{i}-k \int_{q_{i}}^{\hat{x}_{i}+h} x_{i} f\left(x_{i}\right) \mathrm{d} x_{i}\right]-c \sum_{i=1}^{n} q_{i}-e\left(\sum_{i=1}^{n} q_{i}\right)^{2} \\
= & \sum_{i=1}^{n}\left\{\frac{\left(p_{i}-c_{i}-v\right)}{4 h}\left[q_{i}^{2}-\left(\hat{x}_{i}-h\right)^{2}\right]+\frac{v q_{i}}{2 h}\left(q_{i}-\hat{x}_{i}+h\right)+\frac{q_{i}\left(p_{i}-c_{i}+k\right)}{2 h}\left(\hat{x}_{i}+h-q_{i}\right)-\frac{k}{4 h}\left[\left(\hat{x}_{i}+h\right)^{2}-q_{i}^{2}\right]\right\}-c \sum_{i=1}^{n} q_{i}-e\left(\sum_{i=1}^{n} q_{i}\right)^{2} .
\end{aligned}
$$

The detail of equation (9) is as follows:

$$
\begin{aligned}
\pi_{i}^{d 1} & =\pi_{i}-O_{i}(\vec{p}, \vec{q})-U_{i}(\vec{p}, \vec{q}) \\
& =\int_{\hat{x}_{i}-h}^{q_{i}}\left(p_{i}-w-c_{i}\right) x_{i} f\left(x_{i}\right) \mathrm{d} x_{i}+\int_{q_{i}}^{\hat{x}_{i}+h}\left(p_{i}-w-c_{i}\right) q_{i} f\left(x_{i}\right) \mathrm{d} x_{i}-\int_{\hat{x}_{i}-h}^{q_{i}}(w-v)\left(q_{i}-x_{i}\right) f\left(x_{i}\right) \mathrm{d} x_{i}-\int_{q_{i}}^{\hat{x}_{i}+h} k\left(x_{i}-q_{i}\right) f\left(x_{i}\right) \mathrm{d} x_{i} \\
& =\int_{\hat{x}_{i}-h}^{q_{i}}\left(p_{i}-c_{i}-v\right) x_{i} f\left(x_{i}\right) \mathrm{d} x_{i}+v q_{i} \int_{\hat{x}_{i}-h}^{q_{i}} x_{i} f\left(x_{i}\right) \mathrm{d} x_{i}+\int_{q_{i}}^{\hat{x}_{i}+h}\left(p_{i}-c_{i}+k\right) q_{i} f\left(x_{i}\right) \mathrm{d} x_{i}-k \int_{q_{i}}^{\hat{x}_{i}+h} x_{i} f\left(x_{i}\right) \mathrm{d} x_{i}-w q_{i} \\
& =\frac{\left(p_{i}-c_{i}-v\right)}{4 h}\left[q_{i}^{2}-\left(\hat{x}_{i}-h\right)^{2}\right]+\frac{v q_{i}}{2 h}\left(q_{i}-\hat{x}_{i}+h\right)+\frac{q_{i}\left(p_{i}-c_{i}+k\right)}{2 h}\left(\hat{x}_{i}+h-q_{i}\right)-\frac{k}{4 h}\left[\left(\hat{x}_{i}+h\right)^{2}-q_{i}^{2}\right]-c q_{i}-2 e q_{i} \sum_{j=1}^{j=n} q_{j} .
\end{aligned}
$$


The detail of equation (11) is as follows:

$$
\begin{aligned}
\pi_{s}^{d 2}= & \underbrace{w}_{i=1} \sum_{\pi_{s}}^{n} q_{i}-\left[c \sum_{i=1}^{n} q_{i}+e\left(\sum_{i=1}^{n} q_{i}\right)^{2}\right]-\sum_{i=1}^{n}\left[O_{i}(\vec{p}, \vec{q})+U_{i}(\vec{p}, \vec{q})\right] \\
= & w \sum_{i=1}^{n} q_{i}-\left[c \sum_{i=1}^{n} q_{i}+e\left(\sum_{i=1}^{n} q_{i}\right)^{2}\right] \\
& -\sum_{i=1}^{n}\left[\int_{\hat{x}_{i}-h}^{q_{i}}(w-v)\left(q_{i}-x_{i}\right) f\left(x_{i}\right) \mathrm{d} x_{i}+\int_{q_{i}}^{\hat{x}_{i}+h} k\left(x_{i}-q_{i}\right) f\left(x_{i}\right) \mathrm{d} x_{i}\right] \\
= & w \sum_{i=1}^{n} q_{i}-\left[c \sum_{i=1}^{n} q_{i}+e\left(\sum_{i=1}^{n} q_{i}\right)^{2}\right] \\
& -\sum_{i=1}^{n}\left\{\frac{(w-v) q_{i}}{2 h}\left(q_{i}-\hat{x}_{i}+h\right)-\frac{(w-v)}{4 h}\left[q_{i}^{2}-\left(\hat{x}_{i}-h\right)^{2}\right]+\frac{k}{4 h}\left[\left(\hat{x}_{i}+h\right)^{2}-q_{i}^{2}\right]-\frac{k q_{i}}{2 h}\left(\hat{x}_{i}+h-q_{i}\right)\right\} .
\end{aligned}
$$

The detail of equation (12) is as follows:

$$
\begin{aligned}
\frac{d \pi_{s}^{d 2}}{d q_{i}} & =w-\left[c+2 e \sum_{j=1}^{n} q_{j}\right]-\left[\frac{(w-v)}{2 h}\left(q_{i}-\hat{x}_{i}+h\right)-\frac{k}{2 h}\left(\hat{x}_{i}+h-q_{i}\right)\right] \\
& =w-\left[c+2 e \sum_{j=1}^{n} q_{j}\right]-\left[\frac{(w-v+k)}{2 h}\left(q_{i}-\hat{x}_{i}\right)+\frac{w-v-k}{2}\right]=0 .
\end{aligned}
$$

The detail of equation (14) is as follows:@

$$
\begin{aligned}
\pi_{i}^{d 2}= & \int_{\hat{x}_{i}-h}^{q_{i}}\left(p_{i}-w-c_{i}\right) x_{i} f\left(x_{i}\right) \mathrm{d} x_{i}+\int_{q_{i}}^{\hat{x}_{i}+h}\left(p_{i}-w-c_{i}\right) q_{i} f\left(x_{i}\right) \mathrm{d} x_{i} \\
= & \frac{\left(p_{i}-w-c_{i}\right)}{4 h}\left[q_{i}^{2}-\left(\hat{x}_{i}-h\right)^{2}\right]+\frac{q_{i}\left(p_{i}-w-c_{i}\right)}{2 h}\left(\hat{x}_{i}+h-q_{i}\right) \\
= & \frac{\left(p_{i}-w-c_{i}\right)}{4 h}\left[-q_{i}^{2}-\left(q_{i}-Q-h\right)^{2}+2 q_{i}\left(q_{i}-Q+h\right)\right] \\
= & \frac{1}{4 h}\left[\frac{\lambda_{i} d+Q-q_{i}}{\alpha+\beta}+\frac{\sum_{i=1}^{n} q_{i}-n Q-d}{(\alpha+\beta)(n-1-(\alpha / \beta))}-\frac{2 h\left(c+2 e \sum_{j=1}^{n} q_{j}\right)+(k-v) Q-h(k+v)}{h-Q}-c_{i}\right] \\
& \cdot\left[-q_{i}^{2}-\left(q_{i}-Q-h\right)^{2}+2 q_{i}\left(q_{i}-Q+h\right)\right] .
\end{aligned}
$$


The detail of equation (18) is as follows:

$$
\begin{aligned}
\pi_{i}^{r f 1}= & \pi_{i}-O_{i}(\vec{p}, \vec{q})-U_{i}(\vec{p}, \vec{q})+\frac{q_{i}}{\sum_{j=1}^{n} q_{j}} \pi_{s}^{r 1} \\
= & \int_{\hat{x}_{i}-h}^{q_{i}}\left(p_{i}-w-c_{i}\right) x_{i} f\left(x_{i}\right) \mathrm{d} x_{i}+\int_{q_{i}}^{\hat{x}_{i}+h}\left(p_{i}-w-c_{i}\right) q_{i} f\left(x_{i}\right) \mathrm{d} x_{i} \\
& -\int_{\hat{x}_{i}-h}^{q_{i}}(w-v)\left(q_{i}-x_{i}\right) f\left(x_{i}\right) \mathrm{d} x_{i}-\int_{q_{i}}^{\hat{x}_{i}+h} k\left(x_{i}-q_{i}\right) f\left(x_{i}\right) \mathrm{d} x_{i}+w q_{i}-\left(c q_{i}+e q_{i} \sum_{j=1}^{n} q_{j}\right) \\
= & \int_{\hat{x}_{i}-h}^{q_{i}}\left(p_{i}-c_{i}-v\right) x_{i} f\left(x_{i}\right) \mathrm{d} x_{i}+v q_{i} \int_{\hat{x}_{i}-h}^{q_{i}} x_{i} f\left(x_{i}\right) \mathrm{d} x_{i}+\int_{q_{i}}^{\hat{x}_{i}+h}\left(p_{i}-c_{i}+k\right) q_{i} f\left(x_{i}\right) \mathrm{d} x_{i} \\
& -k \int_{q_{i}}^{\hat{x}_{i}+h} x_{i} f\left(x_{i}\right) \mathrm{d} x_{i}-\left(c q_{i}+e q_{i} \sum_{j=1}^{n} q_{j}\right) \\
= & \frac{\left(p_{i}-c_{i}-v\right)}{4 h}\left[q_{i}^{2}-\left(\hat{x}_{i}-h\right)^{2}\right]+\frac{v q_{i}}{2 h}\left(q_{i}-\hat{x}_{i}+h\right)+\frac{q_{i}\left(p_{i}-c_{i}+k\right)}{2 h}\left(\hat{x}_{i}+h-q_{i}\right)-\frac{k}{4 h}\left[\left(\hat{x}_{i}+h\right)^{2}-q_{i}^{2}\right]-c q_{i}-e q_{i} \sum_{j=1}^{n} q_{j} .
\end{aligned}
$$

The detail of equation (21) is as follows:

$$
\begin{aligned}
\pi_{i}^{r r 1}= & \pi_{i}-O_{i}(\vec{p}, \vec{q})-U_{i}(\vec{p}, \vec{q})+\gamma_{i} \pi_{s} \\
= & \int_{\hat{x}_{i}-h}^{q_{i}}\left(p_{i}-w-c_{i}\right) x_{i} f\left(x_{i}\right) \mathrm{d} x_{i}+\int_{q_{i}}^{\hat{x}_{i}+h}\left(p_{i}-w-c_{i}\right) q_{i} f\left(x_{i}\right) \mathrm{d} x_{i}-\int_{\hat{x}_{i}-h}^{q_{i}}(w-v)\left(q_{i}-x_{i}\right) f\left(x_{i}\right) \mathrm{d} x_{i} \\
& -\int_{q_{i}}^{\hat{x}_{i}+h} k\left(x_{i}-q_{i}\right) f\left(x_{i}\right) \mathrm{d} x_{i}+\gamma_{i}\left\{w \sum_{i=1}^{n} q_{i}-\left[c \sum_{i=1}^{n} q_{i}+e\left(\sum_{i=1}^{n} q_{i}\right)^{2}\right]\right\} \\
= & \int_{\hat{x}_{i}-h}^{q_{i}}\left(p_{i}-c_{i}-v\right) x_{i} f\left(x_{i}\right) \mathrm{d} x_{i}+v q_{i} \int_{\hat{x}_{i}-h}^{q_{i}} x_{i} f\left(x_{i}\right) \mathrm{d} x_{i}+\int_{q_{i}}^{x_{i}+h}\left(p_{i}-c_{i}+k\right) q_{i} f\left(x_{i}\right) \mathrm{d} x_{i} \\
& -k \int_{q_{i}}^{\hat{x}_{i}+h} x_{i} f\left(x_{i}\right) \mathrm{d} x_{i}-w q_{i}+\gamma_{i}\left\{w \sum_{i=1}^{n} q_{i}-\left[c \sum_{i=1}^{n} q_{i}+e\left(\sum_{i=1}^{n} q_{i}\right)^{2}\right]\right\} \\
= & \frac{\left(p_{i}-c_{i}-v\right)}{4 h}\left[q_{i}^{2}-\left(\hat{x}_{i}-h\right)^{2}\right]+\frac{v q_{i}}{2 h}\left(q_{i}-\hat{x}_{i}+h\right)+\frac{q_{i}\left(p_{i}-c_{i}+k\right)}{2 h}\left(\hat{x}_{i}+h-q_{i}\right)-\frac{k}{4 h}\left[\left(\hat{x}_{i}+h\right)^{2}-q_{i}^{2}\right] \\
& -\left(c+2 e \sum_{j=1}^{n} q_{j}\right) q_{i}+\gamma_{i}\left\{\left(c+2 e \sum_{j=1}^{n} q_{j}\right)_{i=1}^{n} q_{i}-\left[c \sum_{i=1}^{n} q_{i}+e\left(\sum_{i=1}^{n} q_{i}\right)^{2}\right]\right\} . \\
& \left(\begin{array}{c}
c \\
c
\end{array}\right]
\end{aligned}
$$


The detail of equation (27) is as follows:

$$
\begin{aligned}
\pi_{i}^{r f 2}= & \pi_{i}+\frac{q_{i}}{\sum_{j=1}^{n} q_{j}} \pi_{s}^{r 2} \\
= & \int_{\hat{x}_{i}-h}^{q_{i}}\left(p_{i}-w-c_{i}\right) x_{i} f\left(x_{i}\right) \mathrm{d} x_{i}+\int_{q_{i}}^{\hat{x}_{i}+h}\left(p_{i}-w-c_{i}\right) q_{i} f\left(x_{i}\right) \mathrm{d} x_{i}+w q_{i}-\left(c q_{i}+e q_{i} \sum_{j=1}^{n} q_{j}\right) \\
= & \left(p_{i}-w-c_{i}\right) \frac{\left[-q_{i}^{2}-\left(\hat{x}_{i}-h\right)^{2}+2 q_{i}\left(\hat{x}_{i}+h\right)\right]}{4 h}+w q_{i}-\left(c q_{i}+e q_{i} \sum_{j=1}^{n} q_{j}\right) \\
= & \left(\frac{\lambda_{i} d+Q-q_{i}}{\alpha+\beta}+\frac{\sum_{j=1}^{n} q_{j}-n Q-d}{(\alpha+\beta)(n-1-(\alpha / \beta))}-\frac{2 h\left(c+2 e \sum_{j=1}^{n} q_{j}\right)+(k-v) Q-h(k+v)}{h-Q}-c_{i}\right) \\
& \frac{\left[-q_{i}^{2}-\left(q_{i}-Q-h\right)^{2}+2 q_{i}\left(q_{i}-Q+h\right)\right]}{4 h}+w q_{i}-\left(c q_{i}+e q_{i} \sum_{j=1}^{n} q_{j}\right) .
\end{aligned}
$$

The detail of equation (30) is as follows:

$$
\begin{aligned}
\pi_{i}^{r r 2}= & \pi_{i}+\gamma_{i} \pi_{s}^{r 2}=\int_{\hat{x}_{i}-h}^{q_{i}}\left(p_{i}-w-c_{i}\right) x_{i} f\left(x_{i}\right) \mathrm{d} x_{i}+\int_{q_{i}}^{\hat{x}_{i}+h}\left(p_{i}-w-c_{i}\right) q_{i} f\left(x_{i}\right) \mathrm{d} x_{i}+\gamma_{i}\left\{w \sum_{i=1}^{n} q_{i}-\left[c \sum_{i=1}^{n} q_{i}+e\left(\sum_{i=1}^{n} q_{i}\right)^{2}\right]\right\} \\
= & \left(p_{i}-w-c_{i}\right) \frac{\left[-q_{i}^{2}-\left(\hat{x}_{i}-h\right)^{2}+2 q_{i}\left(\hat{x}_{i}+h\right)\right]}{4 h}+\gamma_{i}\left\{w \sum_{i=1}^{n} q_{i}-\left[c \sum_{i=1}^{n} q_{i}+e\left(\sum_{i=1}^{n} q_{i}\right)^{2}\right]\right\} \\
= & \left(\frac{\lambda_{i} d+Q-q_{i}}{\alpha+\beta}+\frac{\sum_{j=1}^{n} q_{j}-n Q-d}{(\alpha+\beta)(n-1-(\alpha / \beta))}-\frac{2 h\left(c+2 e \sum_{j=1}^{n} q_{j}\right)+(k-v) Q-h(k+v)}{h-Q}-c_{i}\right) \\
& \frac{\left[-q_{i}^{2}-\left(q_{i}-Q-h\right)^{2}+2 q_{i}\left(q_{i}-Q+h\right)\right]}{4 h}+\gamma_{i}\left\{w \sum_{i=1}^{n} q_{i}-\left[c \sum_{i=1}^{n} q_{i}+e\left(\sum_{i=1}^{n} q_{i}\right)^{2}\right]\right\} .
\end{aligned}
$$


The detail of equation (34) is as follows:

$$
\begin{aligned}
& \pi_{i}^{r s 1}=\pi_{i}-\frac{q_{i}}{\sum_{j=1}^{n} q_{j}} \sum_{i=1}^{n}\left[O_{i}(\vec{p}, \vec{q})+U_{i}(\vec{p}, \vec{q})\right] \\
& =\int_{\hat{x}_{i}-h}^{q_{i}}\left(p_{i}-w-c_{i}\right) x_{i} f\left(x_{i}\right) \mathrm{d} x_{i}+\int_{q_{i}}^{\hat{x}_{i}+h}\left(p_{i}-w-c_{i}\right) q_{i} f\left(x_{i}\right) \mathrm{d} x_{i} \\
& -\frac{q_{i}}{\sum_{j=1}^{n} q_{j}} \sum_{j=1}^{n}\left[\int_{\hat{x}_{j}-h}^{q_{j}}(w-v)\left(q_{j}-x_{j}\right) f\left(x_{j}\right) \mathrm{d} x_{j}+\int_{q_{j}}^{\hat{x}_{j}+h} k\left(x_{j}-q_{j}\right) f\left(x_{j}\right) \mathrm{d} x_{j}\right] \\
& =\frac{\left(p_{i}-w-c_{i}\right)}{4 h}\left[q_{i}^{2}-\left(\hat{x}_{i}-h\right)^{2}\right]+\frac{q_{i}\left(p_{i}-w-c_{i}\right)}{2 h}\left(\hat{x}_{i}+h-q_{i}\right)-\frac{q_{i}}{\sum_{j=1}^{n} q_{j}} \\
& \sum_{j=1}^{n}\left\{\frac{(w-v) q_{j}}{2 h}\left(q_{j}-\hat{x}_{j}+h\right)-\frac{(w-v)}{4 h}\left[q_{j}^{2}-\left(\hat{x}_{j}-h\right)^{2}\right]+\frac{k}{4 h}\left[\left(\hat{x}_{j}+h\right)^{2}-q_{j}^{2}\right]-\frac{k q_{j}}{2 h}\left(\hat{x}_{j}+h-q_{j}\right)\right\} \\
& =\frac{\left(p_{i}-w-c_{i}\right)}{4 h}\left[q_{i}^{2}-\left(\hat{x}_{i}-h\right)^{2}\right]+\frac{q_{i}\left(p_{i}-w-c_{i}\right)}{2 h}\left(\hat{x}_{i}+h-q_{i}\right)- \\
& \frac{q_{i}}{\sum_{j=1}^{n} q_{j}} \sum_{j=1}^{n}\left\{\frac{(w-v+k)}{4 h} q_{j}^{2}-q_{j}\left[\frac{(w-v)\left(\hat{x}_{j}-h\right)}{2 h}+\frac{k\left(\hat{x}_{j}+h\right)}{2 h}\right]+\frac{(w-v)}{4 h}\left(\hat{x}_{j}-h\right)^{2}+\frac{k}{4 h}\left(\hat{x}_{j}+h\right)^{2}\right\} \\
& =\frac{\left(p_{i}-c-2 e \sum_{j=1}^{n} q_{j}-c_{i}\right)}{4 h}\left[q_{i}^{2}-\left(\hat{x}_{i}-h\right)^{2}\right]+\frac{q_{i}\left(p_{i}-c-2 e \sum_{j=1}^{n} q_{j}-c_{i}\right)}{2 h}\left(\hat{x}_{i}+h-q_{i}\right)-\frac{q_{i}}{\sum_{j=1}^{n} q_{j}} \\
& \sum_{j=1}^{n}\left\{\frac{\left(c+2 e \sum_{j=1}^{n} q_{j}-v+k\right)}{4 h} q_{j}^{2}-q_{j}\left[\frac{\left(c+2 e \sum_{j=1}^{n} q_{j}-v\right)\left(\hat{x}_{j}-h\right)}{2 h}+\frac{k\left(\hat{x}_{j}+h\right)}{2 h}\right]\right. \\
& \left.+\frac{\left(c+2 e \sum_{j=1}^{n} q_{j}-v\right)}{4 h}\left(\hat{x}_{j}-h\right)^{2}+\frac{k}{4 h}\left(\hat{x}_{j}+h\right)^{2}\right\} .
\end{aligned}
$$

The detail of equation (37) is as follows:

$$
\begin{aligned}
\pi_{s}^{r s 2}= & \underbrace{w \sum_{i=1}^{n} q_{i}-\left[c \sum_{i=1}^{n} q_{i}+e\left(\sum_{i=1}^{n} q_{i}\right)^{2}\right]}_{\pi_{s}}-\phi_{0} \sum_{i=1}^{n}\left[O_{i}(\vec{p}, \vec{q})+U_{i}(\vec{p}, \vec{q})\right] \\
= & w \sum_{i=1}^{n} q_{i}-\left[c \sum_{i=1}^{n} q_{i}+e\left(\sum_{i=1}^{n} q_{i}\right)^{2}\right]-\phi_{0} \sum_{i=1}^{n}\left[\int_{\hat{x}_{i}-h}^{q_{i}}(w-v)\left(q_{i}-x_{i}\right) f\left(x_{i}\right) \mathrm{d} x_{i}+\int_{q_{i}}^{\hat{x}_{i}+h} k\left(x_{i}-q_{i}\right) f\left(x_{i}\right) \mathrm{d} x_{i}\right] \\
= & w \sum_{i=1}^{n} q_{i}-\left[c \sum_{i=1}^{n} q_{i}+e\left(\sum_{i=1}^{n} q_{i}\right)^{2}\right] \\
& -\phi_{0} \sum_{i=1}^{n}\left\{\frac{(w-v) q_{i}}{2 h}\left(q_{i}-\hat{x}_{i}+h\right)-\frac{(w-v)}{4 h}\left[q_{i}^{2}-\left(\hat{x}_{i}-h\right)^{2}\right]+\frac{k}{4 h}\left[\left(\hat{x}_{i}+h\right)^{2}-q_{i}^{2}\right]-\frac{k q_{i}}{2 h}\left(\hat{x}_{i}+h-q_{i}\right)\right\}
\end{aligned}
$$


The detail of equation (40) is as follows:

$$
\begin{aligned}
\pi_{i}^{r s 2}= & \pi_{i}-\phi_{i} \sum_{i=1}^{n}\left[O_{i}(\vec{p}, \vec{q})+U_{i}(\vec{p}, t \vec{q})\right]=\int_{\hat{x}_{i}-h}^{q_{i}}\left(p_{i}-w-c_{i}\right) x_{i} f\left(x_{i}\right) \mathrm{d} x_{i}+\int_{q_{i}}^{\hat{x}_{i}+h}\left(p_{i}-w-c_{i}\right) q_{i} f\left(x_{i}\right) \mathrm{d} x_{i} \\
& -\phi_{i} \sum_{j=1}^{n}\left\{\int_{\hat{x}_{j}-h}^{q_{j}}(w-v)\left(q_{j}-x_{j}\right) f\left(x_{j}\right) \mathrm{d} x_{j}+\int_{q_{j}}^{\hat{x}_{j}+h} k\left(x_{j}-q_{j}\right) f\left(x_{j}\right) \mathrm{d} x_{j}\right\} \\
= & \frac{\left(p_{i}-w-c_{i}\right)}{4 h}\left[q_{i}^{2}-\left(\hat{x}_{i}-h\right)^{2}\right]+\frac{q_{i}\left(p_{i}-w-c_{i}\right)}{2 h}\left(\hat{x}_{i}+h-q_{i}\right) \\
& -\phi_{i} \sum_{j=1}^{n}\left\{\frac{(w-v+k)}{4 h} q_{j}^{2}-q_{j}\left[\frac{(w-v)\left(\hat{x}_{j}-h\right)}{2 h}+\frac{k\left(\hat{x}_{j}+h\right)}{2 h}\right]+\frac{(w-v)}{4 h}\left(\hat{x}_{j}-h\right)^{2}+\frac{k}{4 h}\left(\hat{x}_{j}+h\right)^{2}\right\} \\
= & \frac{\left(p_{i}-w-c_{i}\right)}{4 h}\left[q_{i}^{2}-\left(q_{i}-Q-h\right)^{2}\right]+\frac{q_{i}\left(p_{i}-w-c_{i}\right)}{2 h}(h-Q) \\
& -\phi_{i} \sum_{j=1}^{n}\left\{\frac{(w-v+k)}{4 h} q_{j}^{2}-q_{j}\left[\frac{(w-v)\left(q_{j}-Q-h\right)}{2 h}+\frac{k\left(q_{j}-Q+h\right)}{2 h}\right]+\frac{(w-v)}{4 h}\left(q_{j}-Q-h\right)^{2}+\frac{k}{4 h}\left(q_{j}-Q+h\right)^{2}\right\} .
\end{aligned}
$$

The detail of equation (43) is as follows:

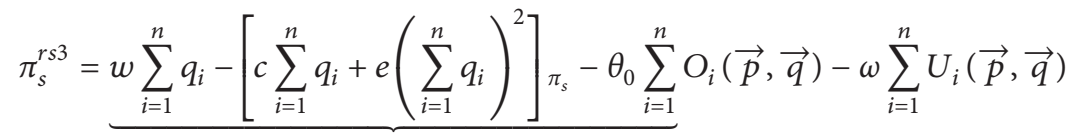

$$
\begin{aligned}
& =w \sum_{i=1}^{n} q_{i}-\left[c \sum_{i=1}^{n} q_{i}+e\left(\sum_{i=1}^{n} q_{i}\right)^{2}\right]-\theta_{0} \sum_{i=1}^{n} \int_{\hat{x}_{i}-h}^{q_{i}}(w-v)\left(q_{i}-x_{i}\right) f\left(x_{i}\right) \mathrm{d} x_{i}-\omega_{0} \sum_{i=1}^{n} \int_{q_{i}}^{\hat{x}_{i}+h} k\left(x_{i}-q_{i}\right) f\left(x_{i}\right) \mathrm{d} x_{i} \\
& =w \sum_{i=1}^{n} q_{i}-\left[c \sum_{i=1}^{n} q_{i}+e\left(\sum_{i=1}^{i=n} q_{i}\right)^{2}\right]-\theta_{0} \sum_{j=1}^{n}\left\{\frac{(w-v) q_{j}}{2 h}\left(q_{j}-\hat{x}_{j}+h\right)-\frac{(w-v)}{4 h}\left[q_{j}^{2}-\left(\hat{x}_{j}-h\right)^{2}\right]\right\} \\
& -\omega_{0} \sum_{j=1}^{n}\left\{\frac{k}{4 h}\left[\left(\hat{x}_{j}+h\right)^{2}-q_{j}^{2}\right]-\frac{k q_{j}}{2 h}\left(\hat{x}_{j}+h-q_{j}\right)\right\} .
\end{aligned}
$$

The detail of equation (46) is as follows: 


$$
\begin{aligned}
\pi_{i}^{r s 3}= & \pi_{i}-\theta_{i} \sum_{i=1}^{n} O_{i}(\vec{p}, \vec{q})-\omega_{i} \sum_{i=1}^{n} U_{i}(\vec{p}, \vec{q})=\int_{\hat{x}_{i}-h}^{q_{i}}\left(p_{i}-w-c_{i}\right) x_{i} f\left(x_{i}\right) \mathrm{d} x_{i}+\int_{q_{i}}^{\hat{x}_{i}+h}\left(p_{i}-w-c_{i}\right) q_{i} f\left(x_{i}\right) \mathrm{d} x_{i} \\
& -\theta_{i} \sum_{i=1}^{n} \int_{\hat{x}_{i}-h}^{q_{i}}(w-v)\left(q_{i}-x_{i}\right) f\left(x_{i}\right) \mathrm{d} x_{i}-\omega_{i} \sum_{i=1}^{n} \int_{q_{i}}^{\hat{x}_{i}+h} k\left(x_{i}-q_{i}\right) f\left(x_{i}\right) \mathrm{d} x_{i} \\
= & \frac{\left(p_{i}-w-c_{i}\right)}{4 h}\left[q_{i}^{2}-\left(\hat{x}_{i}-h\right)^{2}\right]+\frac{q_{i}\left(p_{i}-w-c_{i}\right)}{2 h}\left(\hat{x}_{i}+h-q_{i}\right)-\theta_{i} \sum_{j=1}^{n}\left\{\frac{(w-v) q_{j}}{2 h}\left(q_{j}-\hat{x}_{j}+h\right)-\frac{(w-v)}{4 h}\left[q_{j}^{2}-\left(\hat{x}_{j}-h\right)^{2}\right]\right\} \\
& -\omega_{i} \sum_{j=1}^{n}\left\{\frac{k}{4 h}\left[\left(\hat{x}_{j}+h\right)^{2}-q_{j}^{2}\right]-\frac{k q_{j}}{2 h}\left(\hat{x}_{j}+h-q_{j}\right)\right\} \\
= & \frac{\left(p_{i}-w-c_{i}\right)}{4 h}\left[q_{i}^{2}-\left(q_{i}-Q-h\right)^{2}\right]+\frac{q_{i}\left(p_{i}-w-c_{i}\right)}{2 h}(h-Q)-\theta_{i} \sum_{j=1}^{n}\left\{\frac{(w-v) q_{j}}{2 h}(Q+h)-\frac{(w-v)}{4 h}\left[q_{j}^{2}-\left(q_{j}-Q-h\right)^{2}\right]\right\} \\
& -\omega_{i} \sum_{j=1}^{n}\left\{\frac{k}{4 h}\left[\left(q_{j}-Q+h\right)^{2}-q_{j}^{2}\right]-\frac{k q_{j}}{2 h}(h-Q)\right\} .
\end{aligned}
$$

Proof of Proposition 1. $\forall j \in N, j \neq i, p_{j}=0$, and when $\lambda_{i} d-\alpha p_{i}>0$, we can obtain $0<p_{i}<\left(\lambda_{i} d / \alpha\right)$. Meanwhile, $q_{i} \in\left[\hat{x}_{i}-h, \hat{x}_{i}+h\right]$, so the decision variables are bounded and the continuity conditions are satisfied. According to the axiom of choice and bounded continuity, the maximum value of the decision function can be determined [39, 40].

Solve the first and second derivatives of $\pi_{s c}^{c}$ :

$$
\begin{aligned}
& \frac{\partial \pi_{\mathrm{sc}}^{c}}{\partial p_{i}}=\frac{q_{i}^{2}-\left(\hat{x}_{i}-h\right)^{2}}{4 h}+\frac{\alpha\left(p_{i}-c_{i}-v\right)\left(\hat{x}_{i}-h\right)}{2 h}+\frac{\alpha v q_{i}}{2 h}+\frac{q_{i}\left(\hat{x}_{i}+h-q_{i}\right)}{2 h}-\frac{\alpha q_{i}\left(p_{i}-c_{i}+k\right)}{2 h}+\frac{\alpha k\left(\hat{x}_{i}+h\right)}{2 h} \\
& +\sum_{\substack{j=1 \\
j \neq i}}^{n}\left[-\frac{\beta\left(p_{j}-c_{j}-v\right)\left(\hat{x}_{j}-h\right)}{2 h}+\frac{\beta q_{j}\left(p_{j}-c_{j}+k-v\right)}{2 h}-\frac{\beta k\left(\hat{x}_{j}+h\right)}{2 h}\right] \\
& =-\frac{q_{i}^{2}}{4 h}+\frac{q_{i}}{2 h}\left(\hat{x}_{i}+h+\alpha v-\alpha p_{i}+\alpha c_{i}-\alpha k\right)-\frac{\left(\hat{x}_{i}-h\right)^{2}}{4 h}+\frac{\alpha\left(p_{i}-c_{i}-v\right)\left(\hat{x}_{i}-h\right)}{2 h}+\frac{\alpha k\left(\hat{x}_{i}+h\right)}{2 h} \\
& +\sum_{\substack{j=1 \\
j \neq i}}^{n}\left[-\frac{\beta\left(p_{j}-c_{j}-v\right)\left(\hat{x}_{j}-h\right)}{2 h}+\frac{\beta q_{j}\left(p_{j}-c_{j}+k-v\right)}{2 h}-\frac{\beta k\left(\hat{x}_{j}+h\right)}{2 h}\right] \\
& \frac{\partial^{2} \pi_{\mathrm{sc}}^{c}}{\partial p_{i}^{2}}=-\frac{\alpha q_{i}}{h}+\frac{\alpha\left(\hat{x}_{i}-h\right)}{2 h}+\frac{\alpha\left(\hat{x}_{i}-h\right)}{2 h}-\frac{\alpha^{2}\left(p_{i}-c_{i}-v\right)}{2 h}-\frac{\alpha^{2} k}{2 h}+\sum_{\substack{j=1 \\
j \neq i}}^{n}\left[-\frac{\beta^{2}\left(p_{j}-c_{j}-v+k\right)}{2 h}\right] \\
& =\frac{\alpha\left(\hat{x}_{i}-h-q_{i}\right)}{h}-\frac{\alpha^{2}\left(p_{i}-c_{i}-v+k\right)}{2 h}+\sum_{\substack{j=1 \\
j \neq i}}^{n}\left[-\frac{\beta^{2}\left(p_{j}-c_{j}-v+k\right)}{2 h}\right]<0, \\
& \frac{\partial \pi_{\mathrm{sc}}^{c}}{\partial q_{i}}=\frac{q_{i}\left(p_{i}-c_{i}-v\right)}{2 h}+\frac{v\left(2 q_{i}-\hat{x}_{i}+h\right)}{2 h}+\frac{\left(p_{i}-c_{i}+k\right)\left(\hat{x}_{i}+h-2 q_{i}\right)}{2 h}+\frac{k q_{i}}{2 h}-c-2 e \sum_{j=1}^{n} q_{j} \\
& =\frac{\left(\hat{x}_{i}-q_{i}\right)\left(p_{i}-c_{i}-v+k\right)}{2 h}+\frac{p_{i}-c_{i}+k+v}{2}-c-2 e \sum_{j=1}^{n} q_{j}, \\
& \frac{\partial^{2} \pi_{\mathrm{sc}}^{c}}{\partial q_{i}^{2}}=-\frac{p_{i}-c_{i}-v+k}{2 h}-2 e<0
\end{aligned}
$$


Therefore, $\forall i \in N$, and there is an optimal $\left(p_{i}, q_{i}\right)$ to $\operatorname{maximize} \pi_{\mathrm{sc}}^{c}$.
Proof of Proposition 2. Solve the first and second derivatives of $\pi_{i}^{d 1}$ :

$$
\begin{aligned}
& \frac{\partial \pi_{i}^{d 1}}{\partial p_{i}}=\frac{q_{i}^{2}-\left(\hat{x}_{i}-h\right)^{2}}{4 h}+\frac{\alpha\left(p_{i}-c_{i}-v\right)\left(\hat{x}_{i}-h\right)}{2 h}+\frac{\alpha v q_{i}}{2 h}+\frac{q_{i}\left(\hat{x}_{i}+h-q_{i}\right)}{2 h}-\frac{\alpha q_{i}\left(p_{i}-c_{i}+k\right)}{2 h}+\frac{\alpha k}{2 h}\left(\hat{x}_{i}+h\right) \\
& =-\frac{q_{i}^{2}}{4 h}+\frac{q_{i}\left(\hat{x}_{i}+h\right)}{2 h}-\frac{\alpha q_{i}\left(p_{i}-c_{i}-v+k\right)}{2 h}-\frac{\left(\hat{x}_{i}-h\right)^{2}}{4 h}+\frac{\alpha\left(p_{i}-c_{i}-v\right)\left(\hat{x}_{i}-h\right)}{2 h}+\frac{\alpha k}{2 h}\left(\hat{x}_{i}+h\right), \\
& \frac{\partial^{2} \pi_{i}^{d 1}}{\partial p_{i}^{2}}=-\frac{\alpha q_{i}}{2 h}-\frac{\alpha q_{i}}{2 h}+\frac{\alpha\left(\hat{x}_{i}-h\right)}{2 h}+\frac{\alpha\left(\hat{x}_{i}-h\right)}{2 h}-\frac{\alpha^{2}\left(p_{i}-c_{i}-v\right)}{2 h}-\frac{\alpha^{2} k}{2 h} \\
& =-\frac{\alpha(\overbrace{q_{i}-\hat{x}_{i}+h}^{>0})}{h}-\frac{\alpha^{2}(\overbrace{p_{i}-c_{i}}^{>0} \overbrace{-v+k}^{>0})}{2 h}<0, \\
& \frac{\partial \pi_{i}^{d 1}}{\partial q_{i}}=\frac{q_{i}\left(p_{i}-c_{i}-v\right)}{2 h}+\frac{v\left(2 q_{i}-\hat{x}_{i}+h\right)}{2 h}+\frac{\left(p_{i}-c_{i}+k\right)\left(\hat{x}_{i}+h-2 q_{i}\right)}{2 h}+\frac{k q_{i}}{2 h}-c \\
& -2 e \sum_{j=1}^{n} q_{j}-2 e q_{i}=\frac{\left(p_{i}-c_{i}+k-v\right)\left(\hat{x}_{i}-q_{i}\right)}{2 h}+\frac{p_{i}-c_{i}+k+v}{2}-c-2 e \sum_{j=1}^{n} q_{j}-2 e q_{i}, \\
& \frac{\partial^{2} \pi_{i}^{d 1}}{\partial q_{i}^{2}}=-\frac{\overbrace{p_{i}-c_{i}}^{>0}+\overbrace{k-v}^{>0}}{2 h}-4 e<0
\end{aligned}
$$

Similar to the centralized optimal solution, the implicit expression can be expressed in Proposition 2.
Proof of Proposition 3. Solve the first and second derivatives of $\pi_{i}^{d 2}$ :

$$
\begin{aligned}
\frac{\partial \pi_{i}^{d 2}}{\partial q_{i}} & =p_{i}-w-c_{i}+\frac{-q_{i}^{2}-\left(q_{i}-Q-h\right)^{2}+2 q_{i}\left(q_{i}-Q+h\right)}{4 h} \times\left[-\frac{1}{\alpha+\beta}+\frac{1}{(\alpha+\beta)(n-1-(\alpha / \beta))}-\frac{4 h e}{h-Q}\right] \\
& =\frac{\lambda_{i} d+Q-q_{i}}{\alpha+\beta}+\frac{\sum_{j=1}^{n} q_{j}-n Q-d}{(\alpha+\beta)(n-1-(\alpha / \beta))}-\frac{2 h\left(c+2 e \sum_{j=1}^{n} q_{j}\right)+(k-v) Q-h(k+v)}{h-Q}- \\
& c_{i}+\frac{-q_{i}^{2}-\left(q_{i}-Q-h\right)^{2}+2 q_{i}\left(q_{i}-Q+h\right)}{4 h} \times\left[-\frac{1}{\alpha+\beta}+\frac{1}{(\alpha+\beta)(n-1-(\alpha / \beta))}-\frac{4 h e}{h-Q}\right], \\
\frac{\partial^{2} \pi_{i}^{d 2}}{\partial q_{i}^{2}} & =2[\overbrace{-\frac{1}{\alpha+\beta}}^{<-\frac{<0}{(\alpha+\beta)(n-1-(\alpha / \beta))}}-\overbrace{\frac{4 h e}{h-Q}}^{<0}]<0 .
\end{aligned}
$$


Similar to the centralized optimal solution, the implicit expression can be expressed in Proposition 3.
Proof of Proposition 4. Solve the first and second derivatives of $\pi_{i}^{r f 1}$ :

$$
\begin{aligned}
& \frac{\partial \pi_{i}^{r f 1}}{\partial p_{i}}=-\frac{q_{i}^{2}}{4 h}+\frac{q_{i}\left(\hat{x}_{i}+h\right)}{2 h}-\frac{\alpha q_{i}\left(p_{i}-c_{i}-v+k\right)}{2 h}-\frac{\left(\hat{x}_{i}-h\right)^{2}}{4 h}+\frac{\alpha\left(p_{i}-c_{i}-v\right)\left(\hat{x}_{i}-h\right)}{2 h}+\frac{\alpha k}{2 h}\left(\hat{x}_{i}+h\right), \\
& \frac{\partial^{2} \pi_{i}^{r f 1}}{\partial p_{i}^{2}}=\frac{\alpha(\overbrace{q_{i}-\hat{x}_{i}+h}^{>0})}{h}-\frac{\alpha^{2}(\overbrace{p_{i}-c_{i}}^{>0} \overbrace{-v+k}^{>0})}{2 h}<0, \\
& \frac{\partial \pi_{i}^{r f 1}}{\partial q_{i}}=\frac{\left(p_{i}-c_{i}+k-v\right)\left(\hat{x}_{i}-q_{i}\right)}{2 h}+\frac{p_{i}-c_{i}+k+v}{2}-c-e \sum_{j=1}^{n} q_{j}-e q_{i}, \\
& \frac{\partial^{2} \pi_{i}^{r f 1}}{\partial q_{i}^{2}}=-\frac{\overbrace{p_{i}-c_{i}}^{>0}+\overbrace{k-v}^{>0}}{2 h}-2 e<0
\end{aligned}
$$

Similar to the centralized optimal solution, the implicit expression can be expressed in Proposition 4.
Proof of Proposition 5. Solve the first and second derivatives of $\pi_{i}^{r r 1}$ :

$$
\begin{aligned}
& \frac{\partial \pi_{i}^{r r 1}}{\partial p_{i}}=-\frac{q_{i}^{2}}{4 h}+\frac{q_{i}\left(\hat{x}_{i}+h\right)}{2 h}-\frac{\alpha q_{i}\left(p_{i}-c_{i}-v+k\right)}{2 h}-\frac{\left(\hat{x}_{i}-h\right)^{2}}{4 h}+\frac{\alpha\left(p_{i}-c_{i}-v\right)\left(\hat{x}_{i}-h\right)}{2 h}+\frac{\alpha k}{2 h}\left(\hat{x}_{i}+h\right), \\
& \frac{\partial^{2} \pi_{i}^{r r 1}}{\partial p_{i}^{2}}=-\frac{\alpha(\overbrace{q_{i}-\hat{x}_{i}+h}^{>0})}{h}-\frac{\alpha^{2}(\overbrace{p_{i}-c_{i}}^{>0} \overbrace{-v+k}^{>0})}{2 h}<0, \\
& \frac{\partial \pi_{i}^{r r 1}}{\partial q_{i}}=\frac{\left(p_{i}-c_{i}+k-v\right)\left(\hat{x}_{i}-q_{i}\right)}{2 h}+\frac{p_{i}-c_{i}+k+v}{2}-c-2 e \sum_{j=1}^{n} q_{j}-2 e q_{i}+2 \gamma_{i} e \sum_{j=1}^{n} q_{j}, \\
& \frac{\partial^{2} \pi_{i}^{r r 1}}{\partial q_{i}^{2}}=-\frac{\overbrace{p_{i}-c_{i}}^{>0}+\overbrace{k-v}^{>0}}{2 h} \overbrace{-4 e+2 \gamma_{i} e}^{<0}<0 .
\end{aligned}
$$


Similar to the centralized optimal solution, the implicit expression can be expressed in Proposition 5.
Proof of Proposition 6. Solve the first and second derivatives of $\pi_{i}^{r f 2}$ :

$$
\begin{aligned}
\frac{\partial \pi_{i}^{r f 2}}{\partial q_{i}}= & \left(-\frac{1}{\alpha+\beta}+\frac{1}{(\alpha+\beta)(n-1-(\alpha / \beta))}-\frac{4 h e}{h-Q}\right) \frac{\left[-q_{i}^{2}-\left(q_{i}-Q-h\right)^{2}+2 q_{i}\left(q_{i}-Q+h\right)\right]}{4 h} \\
& +\left(\frac{\lambda_{i} d+Q-q_{i}}{\alpha+\beta}+\frac{\sum_{j=1}^{n} q_{j}-n Q-d}{(\alpha+\beta)(n-1-(\alpha / \beta))}-\frac{2 h\left(c+2 e \sum_{j=1}^{n} q_{j}\right)+(k-v) Q-h(k+v)}{h-Q}-c_{i}\right) \\
& +\frac{2 h\left(c+2 e \sum_{j=1}^{n} q_{j}\right)+(k-v) Q-h(k+v)}{h-Q}+\frac{4 h e}{h-Q} q_{i}-\left(c+e q_{i}+e \sum_{j=1}^{n} q_{j}\right), \\
\frac{\partial^{2} \pi_{i}^{r f 2}}{\partial q_{i}^{2}}= & 2[\overbrace{-\frac{1}{\alpha+\beta}}^{<-0} \frac{1}{(\alpha+\beta)(n-1-(\alpha / \beta))}]-2 e<0 .
\end{aligned}
$$

Similar to the centralized optimal solution, the implicit expression can be expressed in Proposition 6.
Proof of Proposition 7. Solve the first and second derivatives of $\pi_{i}^{r r 2}$ :

$$
\begin{aligned}
\frac{\partial \pi_{i}^{r r 2}}{\partial q_{i}}= & \left(-\frac{1}{\alpha+\beta}+\frac{1}{(\alpha+\beta)(n-1-(\alpha / \beta))}-\frac{4 h e}{h-Q}\right) \frac{\left[-q_{i}^{2}-\left(q_{i}-Q-h\right)^{2}+2 q_{i}\left(q_{i}-Q+h\right)\right]}{4 h} \\
& +\left(\frac{\lambda_{i} d+Q-q_{i}}{\alpha+\beta}+\frac{\sum_{j=1}^{n} q_{j}-n Q-d}{(\alpha+\beta)(n-1-(\alpha / \beta))}-\frac{2 h\left(c+2 e \sum_{j=1}^{n} q_{j}\right)+(k-v) Q-h(k+v)}{h-Q}-c_{i}\right) \\
& +\gamma_{i} \frac{2 h\left(c+2 e \sum_{j=1}^{n} q_{j}\right)+(k-v) Q-h(k+v)}{h-Q}+\gamma_{i} \frac{4 h e}{h-Q} \sum_{i=1}^{n} q_{i}-\gamma_{i}\left(c+2 e \sum_{j=1}^{n} q_{j}\right), \\
\frac{\partial^{2} \pi_{i}^{r r 2}}{\partial q_{i}^{2}}= & 2[\overbrace{-\frac{1}{\alpha+\beta}}^{<-\frac{1}{(\alpha+\beta)(n-1-(\alpha / \beta))}}]+\overbrace{\left(\gamma_{i}-1\right) \frac{8 h e}{h-Q}}^{<-2 \gamma_{i} e}<0 .
\end{aligned}
$$

Similar to the centralized optimal solution, the implicit expression can be expressed in Proposition 7.
Proof of Proposition 8. Solve the first and second derivatives of $\pi_{i}^{r s 1}$ : 


$$
\begin{aligned}
& \frac{\partial \pi_{i}^{r s 2}}{\partial q_{i}}=-\frac{e}{2 h}\left[q_{i}^{2}-\left(\hat{x}_{i}-h\right)^{2}\right]+\frac{\left(p_{i}-c-2 e \sum_{j=1}^{n} q_{j}-c_{i}\right)}{2 h}\left(\hat{x}_{i}+h-q_{i}\right)-\frac{e q_{i}}{h}\left(\hat{x}_{i}+h-q_{i}\right)- \\
& \frac{\sum_{j=1}^{n} q_{j}-q_{i}}{\sum_{j=1}^{n} q_{j}} \sum_{j=1}^{n}\left\{\frac{\left(c+2 e \sum_{j=1}^{n} q_{j}-v+k\right)}{4 h} q_{j}^{2}-q_{j}\left[\frac{\left(c+2 e \sum_{j=1}^{n} q_{j}-v\right)\left(\hat{x}_{j}-h\right)}{2 h}+\frac{k\left(\hat{x}_{j}-h\right)}{2 h}\right]\right. \\
& \left.+\frac{\left(c+2 e \sum_{j=1}^{n} q_{j}-v\right)}{4 h}\left(\hat{x}_{j}-h\right)^{2}+\frac{k}{4 h}\left(\hat{x}_{j}-h\right)^{2}\right\} \\
& -\frac{q_{i}}{\sum_{j=1}^{n} q_{j}}\left\{\sum_{j=1}^{n}\left[\frac{e}{2 h} q_{j}^{2}-\frac{q_{j} e\left(\hat{x}_{j}-h\right)}{h}+\frac{e}{2 h}\left(\hat{x}_{j}-h\right)^{2}\right]+\frac{c+2 e \sum_{j=1}^{n} q_{j}-v+k}{2 h} q_{i}\right. \\
& \left.-\frac{\left(c+2 e \sum_{j=1}^{n} q_{j}-v\right)\left(\hat{x}_{i}-h\right)}{2 h}-\frac{k\left(\hat{x}_{i}-h\right)}{2 h}\right\} \\
& \frac{\partial \pi_{i}^{r s 1}}{\partial q_{i}^{2}}=\overbrace{-\frac{2 e}{h}\left(\hat{x}_{i}+h-q_{i}\right)}^{>0}-\overbrace{\frac{p_{i}-c-2 e \sum_{j=1}^{n} q_{j}-c_{i}}{2 h}}^{>0}-\overbrace{\frac{2 \sum_{j=1}^{n} q_{j}\left(\sum_{j=1}^{n} q_{j}-q_{i}\right)}{\left(\sum_{j=1}^{n} q_{j}\right)^{4}}}^{>0} \\
& \times \sum_{j=1}^{n}\left\{\frac{\left(c+2 e \sum_{j=1}^{n} q_{j}-v+k\right)}{4 h} q_{j}^{2}-q_{j}\left[\frac{\left(c+2 e \sum_{j=1}^{n} q_{j}-v\right)\left(\hat{x}_{j}-h\right)}{2 h}\right]\right\}+\frac{\left(c+2 e \sum_{j=1}^{n} q_{j}-v\right)}{4 h}\left(\hat{x}_{j}-h\right)^{2} \frac{k}{4 h}\left(\hat{x}_{j}-h\right)^{2} \\
& -2 \frac{\overbrace{\sum_{j=1}^{n} q_{j}-q_{i}}^{>0}}{\sum_{j=1}^{n} q_{j}}\left\{\sum_{j=1}^{n}\left[\frac{e}{2 h} q_{j}^{2}-\frac{q_{j} e\left(\hat{x}_{j}-h\right)}{h}+\frac{e}{2 h}\left(\hat{x}_{j}-h\right)^{2}\right]+\frac{\left(c+2 e \sum_{j=1}^{n} q_{j}-v+k\right)}{2 h} q_{i}-\frac{\left(c+2 e \sum_{j=1}^{n} q_{j}-v\right)\left(\hat{x}_{j}-h\right)}{2 h}\right\} \\
& -\frac{q_{i}}{\sum_{j=1}^{n} q_{j}}\left[\frac{2 e}{h} q_{i}=\frac{2 e\left(\hat{x}_{j}-h\right)}{h}+\frac{c+2 e \sum_{j=1}^{n} q_{j}-v+k}{2 h}\right]<0 \\
& \frac{\partial \pi_{i}^{r s 1}}{\partial p_{i}}=\frac{1}{4 h}\left[q_{i}^{2}-\left(\hat{x}_{i}-h\right)^{2}\right]+\frac{\alpha\left(p_{i}-c-2 e \sum_{j=1}^{n} q_{j}-c_{i}\right)}{2 h}\left(\hat{x}_{i}-h-q_{i}\right)+\frac{q_{i}}{2 h}\left(\hat{x}_{i}+h-q_{i}\right)
\end{aligned}
$$

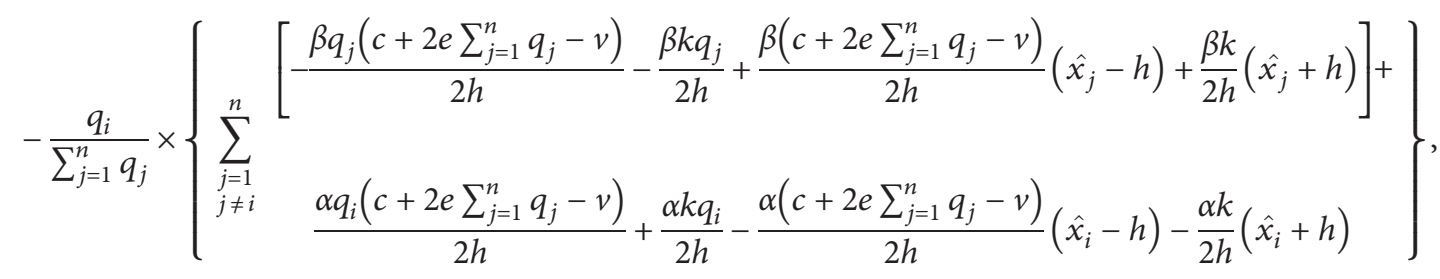

$$
\begin{aligned}
& \frac{\partial^{2} \pi_{i}^{r s 1}}{\partial p_{i}^{2}}=\frac{\alpha}{h}\left(\hat{x}_{i}-h-q_{i}\right)-\frac{\alpha^{2}\left(p_{i}-c-2 e \sum_{j=1}^{n} q_{j}-c_{i}\right)}{2 h} \\
& -\frac{q_{i}}{\sum_{j=1}^{n} q_{j}} \times\left[\frac{(n-1) \beta^{2}\left(c+2 e \sum_{j=1}^{n} q_{j}-v+k\right)}{2 h}+\frac{\alpha^{2}\left(c+2 e \sum_{j=1}^{n} q_{j}-v+k\right)}{2 h}\right]<0
\end{aligned}
$$

Similar to the centralized optimal solution, the implicit expression can be expressed in Proposition 8.
Proof of Proposition 9. Solve the first and second derivatives of $\pi_{i}^{r s 2}$ : 


$$
\begin{aligned}
& \frac{\partial \pi_{i}^{r s 2}}{\partial q_{i}}=p_{i}-w-c_{i}+\frac{q_{i}^{2}-\left(q_{i}-Q-h\right)^{2}+2(h-Q) q_{i}}{4 h} \\
& \times\left[-\frac{1}{\alpha+\beta}+\frac{1}{(\alpha+\beta)(n-1-(\alpha / \beta))}-\frac{4 h e}{\left(2-\phi_{0}\right) h-\phi_{0} Q}\right]-\phi_{i}\left[\frac{(h+Q)^{2}}{4 h} \times \frac{4 n h e}{\left(2-\phi_{0}\right) h-\phi_{0} Q}+\frac{k}{2 h}+\frac{k\left(2 q_{i}-Q+h\right)}{2 h}\right] \\
& =\frac{\lambda_{i} d+Q-q_{i}}{\alpha+\beta}+\frac{\sum_{i=1}^{n} q_{i}-n Q-d}{(\alpha+\beta)(n-1-(\alpha / \beta))}-\frac{2 h\left(c+2 e \sum_{j=1}^{n} q_{j}\right)+\phi_{0}(k-v) Q-\phi_{0} h(k+v)}{\left(2-\phi_{0}\right) h-\phi_{0} Q}-c_{i} \\
& +\frac{q_{i}^{2}-\left(q_{i}-Q-h\right)^{2}+2(h-Q) q_{i}}{4 h} \\
& \times\left[-\frac{1}{\alpha+\beta}+\frac{1}{(\alpha+\beta)(n-1-(\alpha / \beta))}-\frac{4 h e}{\left(2-\phi_{0}\right) h-\phi_{0} Q}\right]-\phi_{i}\left[\frac{(h+Q)^{2}}{4 h} \times \frac{4 n h e}{\left(2-\phi_{0}\right) h-\phi_{0} Q}+\frac{k}{2 h}+\frac{k\left(2 q_{i}-Q+h\right)}{2 h}\right] \\
& \frac{\partial^{2} \pi_{i}^{r s 2}}{\partial q_{i}^{2}}=2[\overbrace{-\frac{1}{\alpha+\beta}}^{<0}+\overbrace{\frac{1}{(\alpha+\beta)(n-1-(\alpha / \beta))}}^{<0}-\overbrace{\frac{4 h e}{\left(2-\phi_{0}\right) h-\phi_{0} Q}}^{<0}]-\phi_{i} \frac{k}{h}<0 .
\end{aligned}
$$

Similar to the centralized optimal solution, the implicit expression can be expressed in Proposition 9.
Proof of Proposition 10. Solve the first and second derivatives of $\pi_{i}^{r s 3}$ :

$$
\begin{aligned}
\frac{\partial \pi_{i}^{r s 3}}{\partial q_{i}}= & p_{i}-w-c_{i}+\frac{q_{i}^{2}-\left(q_{i}-Q-h\right)^{2}+2(h-Q) q_{i}}{4 h} \\
& \times\left[-\frac{1}{\alpha+\beta}+\frac{1}{(\alpha+\beta)(n-1-(\alpha / \beta))}-\frac{4 h e}{\left(2-\theta_{0}\right) h-\theta_{0} Q}\right]-\theta_{i} \times \frac{(Q+h)^{2}}{4 h} \times \frac{4 n h e}{\left(2-\theta_{0}\right) h-\theta_{0} Q} \\
= & \frac{\lambda_{i} d+Q-q_{i}}{\alpha+\beta}+\frac{\sum_{i=1}^{n} q_{i}-n Q-d}{(\alpha+\beta)(n-1-(\alpha / \beta))}-\frac{2 h\left(c+2 e \sum_{j=1}^{n} q_{j}\right)-\theta_{0} v(Q+h)-\omega_{0} k(h-Q)}{\left(2-\theta_{0}\right) h-\theta_{0} Q} \\
& -c_{i}+\frac{q_{i}^{2}-\left(q_{i}-Q-h\right)^{2}+2(h-Q) q_{i}}{4 h} \\
& \times\left[-\frac{1}{\alpha+\beta}+\frac{1}{(\alpha+\beta)(n-1-(\alpha / \beta))}-\frac{4 h e}{\left(2-\theta_{0}\right) h-\theta_{0} Q}\right]-\theta_{i} \times \frac{(Q+h)^{2}}{4 h} \times \frac{4 n h e}{\left(2-\theta_{0}\right) h-\theta_{0} Q}, \\
\frac{\partial^{2} \pi_{i}^{r s 3}}{\partial q_{i}^{2}}= & 3\left[-\frac{<0}{\alpha+\beta}+\frac{<}{\frac{<0}{(\alpha+\beta)(n-1-(\alpha / \beta))}}-\frac{\underbrace{<0}}{\left(2-\theta_{0}\right) h-\theta_{0} Q}\right]<0 .
\end{aligned}
$$

Similar to the centralized optimal solution, the implicit expression can be expressed in Proposition 10. 


\section{Data Availability}

The data used to support the findings of this study are available from the corresponding author.

\section{Conflicts of Interest}

The authors declare that there are no conflicts of interest.

\section{Acknowledgments}

This research was supported by the Fundamental Research Funds for the Central Universities (JBK1507105) and Key Research and Development Program of Hunan Province of China (Grant nos. 2017SK2345 and 2018GK2073).

\section{References}

[1] R. Shen, "National information center: sharing economy GDP is expected to account for $10 \%$ in 2020," 2017.

[2] W. Liu, C. Liu, and M. Ge, "An order allocation model for the two-echelon logistics service supply chain based on cumulative prospect theory," Journal of Purchasing and Supply Management, vol. 19, no. 1, pp. 39-48, 2013.

[3] W. Liu, Q. Wang, Q. Mao, S. Wang, and D. Zhu, "A scheduling model of logistics service supply chain based on the mass customization service and uncertainty of FLSP's operation time," Transportation Research Part E: Logistics and Transportation Review, vol. 83, pp. 189-215, 2015.

[4] W. Liu, Z. Liang, Z. Ye, and L. Liu, "The optimal decision of customer order decoupling point for order insertion scheduling in logistics service supply chain," International Journal of Production Economics, vol. 175, pp. 50-60, 2016.

[5] W. Liu, Y. Liu, D. Zhu, Y. Wang, and Z. Liang, "The influences of demand disruption on logistics service supply chain coordination: a comparison of three coordination modes," International Journal of Production Economics, vol. 179, pp. 59-76, 2016.

[6] W.-H. Liu, X.-C. Xu, and A. Kouhpaenejad, "Deterministic approach to the fairest revenue-sharing coefficient in logistics service supply chain under the stochastic demand condition," Computers \& Industrial Engineering, vol. 66, no. 1, pp. 41-52, 2013.

[7] W. Liu, D. Wang, X. Shen, X. Yan, and W. Wei, "The impacts of distributional and peer-induced fairness concerns on the decision-making of order allocation in logistics service supply chain," Transportation Research Part E: Logistics and Transportation Review, vol. 116, pp. 102-122, 2018.

[8] W. Liu, R. Wu, Z. Liang, and D. Zhu, "Decision model for the customer order decoupling point considering order insertion scheduling with capacity and time constraints in logistics service supply chain," Applied Mathematical Modelling, vol. 54, pp. 112-135, 2018.

[9] W. Liu, M. Wang, D. Zhu, and L. Zhou, "Service capacity procurement of logistics service supply chain with demand updating and loss-averse preference," Applied Mathematical Modelling, vol. 66, pp. 486-507, 2019.

[10] B. Shen, X. Xu, and S. Guo, "The impacts of logistics services on short life cycle products in a global supply chain," Transportation Research Part E: Logistics and Transportation Review, vol. 131, pp. 153-167, 2019.

[11] G. Wang, X. Hu, X. Li, Y. Zhang, S. Feng, and A. Yang, "Multiobjective decisions for provider selection and order allocation considering the position of the CODP in a logistics service supply chain," Computers \& Industrial Engineering, vol. 140, 2020.

[12] C. Narasimhan, P. Papatla, B. Jiang et al., "Sharing economy: review of current research and future directions," Customer Needs and Solutions, vol. 5, no. 1-2, pp. 93-106, 2018.

[13] B. Jiang and L. Tian, "Collaborative consumption: strategic and economic implications of product sharing," Management Science, vol. 64, no. 3, pp. 1171-1188, 2018.

[14] A. Faghih-Imani, R. Hampshire, and L. Marla, "An empirical analysis of bike sharing usage and rebalancing: evidence from Barcelona and Seville," Transportation Research Part A: Policy and Practice, vol. 97, pp. 177-191, 2017.

[15] L. Caggiani, R. Camporeale, M. Ottomanelli, and W. Y. Szeto, "A modeling framework for the dynamic management of freefloating bike-sharing systems," Transportation Research Part C: Emerging Technologies, vol. 87, pp. 159-182, 2018.

[16] I. Eluru and A. Ribeiro, "Bike-sharing stations: a maximal covering location approach," Transportation Research Part A: Policy and Practice, vol. 82, pp. 216-227, 2015.

[17] G. P. Cachon and M. A. Lariviere, "Supply chain coordination with revenue-sharing contracts: strengths and limitations," Management Science, vol. 51, no. 1, pp. 30-44, 2005.

[18] S. Li, Z. Zhu, and L. Huang, "Supply chain coordination and decision making under consignment contract with revenue sharing," International Journal of Production Economics, vol. 120, no. 1, pp. 88-99, 2009.

[19] K. Pan, K. K. Lai, S. C. H. Leung, and D. Xiao, "Revenuesharing versus wholesale price mechanisms under different channel power structures," European Journal of Operational Research, vol. 203, no. 2, pp. 532-538, 2010.

[20] A. Kimms and D. Çetiner, "Approximate nucleolus-based revenue sharing in airline alliances," European Journal of Operational Research, vol. 220, no. 2, pp. 510-521, 2012.

[21] B. Van der Rhee, G. Schmidt, J. A. van der Veen, and V. Venugopal, "Revenue-sharing contracts across an extended supply chain,” Business Horizons, vol. 57, no. 4, pp. 473-482, 2014.

[22] J. Zhang, G. Liu, Q. Zhang, and Z. Bai, "Coordinating a supply chain for deteriorating items with a revenue sharing and cooperative investment contract," Omega, vol. 56, pp. 37-49, 2015.

[23] Q. Wei, S. Li, X. Gou, and B. Huo, "Joint optimal decision of the shared distribution system through revenue-sharing and cooperative investment contracts," Industrial Management \& Data Systems, vol. 119, no. 3, pp. 578-612, 2019.

[24] Q. Zhu, X. Li, and S. Zhao, "Cost-sharing models for green product production and marketing in a food supply chain," Industrial Management \& Data Systems, vol. 118, no. 4, pp. 654-682, 2018.

[25] P. He, Y. He, C. Shi, H. Xu, and L. Zhou, "Cost-sharing contract design in a low-carbon service supply chain," Computers \& Industrial Engineering, vol. 139, 2020.

[26] Y. He and X. Zhao, "Contracts and coordination: supply chains with uncertain demand and supply," Naval Research Logistics (NRL), vol. 63, no. 4, pp. 305-319, 2016.

[27] Q. Bai, M. Chen, and L. Xu, "Revenue and promotional costsharing contract versus two-part tariff contract in coordinating sustainable supply chain systems with deteriorating items," International Journal of Production Economics, vol. 187, pp. 85-101, 2017.

[28] B. $\mathrm{Hu}, \mathrm{D} . \mathrm{Xu}$, and C. Meng, "Inconsistency of a retailer's optimal policies and channel performance under revenue 
sharing contracts," International Journal of Production Economics, vol. 183, pp. 53-65, 2017.

[29] H. Yang, J. Luo, and H. Wang, "The role of revenue sharing and first-mover advantage in emission abatement with carbon tax and consumer environmental awareness," International Journal of Production Economics, vol. 193, pp. 691-702, 2017.

[30] H. Song and X. Gao, "Green supply chain game model and analysis under revenue-sharing contract," Journal of Cleaner Production, vol. 170, pp. 183-192, 2018.

[31] H. Yang and W. Chen, "Retailer-driven carbon emission abatement with consumer environmental awareness and carbon tax: revenue-sharing versus cost-sharing," Omega, vol. 78, pp. 179-191, 2018.

[32] T. Li, R. Zhang, S. Zhao, and B. Liu, "Low carbon strategy analysis under revenue-sharing and cost-sharing contracts," Journal of Cleaner Production, vol. 212, pp. 1462-1477, 2019.

[33] B. Yu, J. Wang, X. Lu, and H. Yang, "Collaboration in a lowcarbon supply chain with reference emission and cost learning effects: cost sharing versus revenue sharing strategies," Journal of Cleaner Production, vol. 250, 2020.

[34] Y. He, H. Huang, and D. Li, "Inventory and pricing decisions for a dual-channel supply chain with deteriorating products," Operational Research, vol. 20, no. 3, pp. 1461-1503, 2020.

[35] D. Xiao, J. Wang, and Q. Lu, "Stimulating sustainability investment level of suppliers with strategic commitment to price and cost sharing in supply chain," Journal of Cleaner Production, vol. 252, 2020.

[36] Y. Ma, N. Wang, Z. He, J. Lu, and H. Liang, “Analysis of the bullwhip effect in two parallel supply chains with interacting price-sensitive demands," European Journal of Operational Research, vol. 243, no. 3, pp. 815-825, 2015.

[37] L. L. Selwyn, Economies of Scale in Computer Usage: Initial Tests and Implications for the Computer Utility, Sloan School of Management, MIT, Cambridge, Massachusetts, 1970.

[38] I. W. Cotton, "Microeconomics and the market for computer services," ACM Computing Surveys, vol. 7, no. 2, pp. 95-111, 1975.

[39] N. C. Petruzzi and M. Dada, "Pricing and the newsvendor problem: a review with extensions," Operations Research, vol. 47, no. 2, pp. 183-194, 1999.

[40] Y. Wang, L. Jiang, and Z.-J. Shen, "Channel performance under consignment contract with revenue sharing," Management Science, vol. 50, no. 1, pp. 34-47, 2004. 